\title{
Energy-Efficient Dynamic Resource Allocation for Opportunistic-Relaying-Assisted SC-FDMA Using Turbo-Equalizer-Aided Soft Decode-and-Forward
}

\author{
Jiayi Zhang, Student Member, IEEE, Lie-Liang Yang, Senior Member, IEEE, and Lajos Hanzo, Fellow, IEEE
}

\begin{abstract}
In this paper, we exploit the benefits of the diversity gains that arise from a cluster of opportunistic relays (ORs) and from the independently fading subcarriers of multiple users. Our goal is to improve the energy efficiency of the decodeand-forward-based OR-assisted single-carrier frequency-division multiple-access (SC-FDMA) uplink, where the direct transmission (DT) link is unavailable. We proposed two joint dynamic resource allocation (JDRA) schemes by assuming that the pilotaided channel-quality information (CQI) of all the users may be exchanged. Furthermore, we take the following two main aspects into account: 1) "first-hop quality awareness" (FHQA) for JDRA-aided OR and 2) "buffering delay awareness" in the context of interleaver-aided channel-coded systems. In addition, frequency-domain turbo equalizers are employed in both the relay and BS's receivers. Our results demonstrate that, compared to the DT benchmarker, the proposed FHQA JDRA schemes can achieve an energy reduction gain of $91 \%$ for a single-antenna base station (BS) receiver and up to $7.4 \%$ for a multiantenna BS when considering the energy consumption due to CQI exchange among relays.
\end{abstract}

Index Terms-Cooperative communications, decode and forward (DF), energy efficiency, frequency-domain turbo equalization, opportunistic relaying (OR), relay selection, single-carrier frequency-division multiple access (SC-FDMA), subcarrier allocation.

\section{INTRODUCTION}

$\mathbf{R}$ ELAY-ASSISTED wireless communications have been explored for diverse ad hoc and cellular networks to improve the attainable spectral or energy efficiency of classic direct transmissions (DTs) [1]. Naturally, the availability of inactive mobiles as candidate relays has the potential to mitigate

Manuscript received October 25, 2011; revised April 30, 2012 and August 8, 2012; accepted September 16, 2012. Date of current version January 14,2013 . This work was supported in part by the Virtual Centre of Excellence in Mobile and Personal Communications (Mobile VCE; www.mobilevce.com) through the Core 5 Research Programme and by the Engineering and Physical Sciences Research Council. Fully detailed technical reports on this paper are available to Industrial Members of Mobile VCE. This paper was presented in part at the IEEE International Conference on Communications, Ottawa, ON, Canada, June 10-15, 2012. The review of this paper was coordinated by Prof. Y. Su.

J. Zhang was with the School of Electronics and Computer Science, University of Southampton, SO17 1BJ Southampton, U.K. He is now with the Bell Labs, Alcatel-Lucent Shanghai Bell, Shanghai 201206, China (e-mail: jiayi.zhang@ieee.org).

L.-L. Yang and L. Hanzo are with the School of Electronics and Computer Science, University of Southampton, SO17 1BJ Southampton, U.K. (e-mail: 1ly@ecs.soton.ac.uk; lh@ecs.soton.ac.uk).

Digital Object Identifier 10.1109/TVT.2012.2220162 the effect of fading. The activation of multiple relays results in cooperative diversity. When dynamically reassigning the relays based on their location or channel quality, we arrive at the concept of opportunistic relaying (OR) [2].

Furthermore, orthogonal frequency-division multiplexing (OFDM)-style broadband frequency-division multiple-access (FDMA) systems, such as the Third-Generation Partnership Project Long Term Evolution (3GPP LTE) system's uplink single-carrier frequency-division multiple-access (SC-FDMA) scheme [3] and orthogonal frequency-division multiple-access (OFDMA) [4], conveniently facilitate near-instantaneous adaptive subband/subcarrier allocation and multiuser (MU) scheduling. This is achieved by exploiting the knowledge of the time-varying channel-state information (CSI) of the subbands/ subcarriers [5]-[9] when communicating over frequencyselective fading channels.

Clearly, directly adopting the conventional OR concept in [2] to OFDM-based systems at the symbol level cannot exploit the time-varying CSI of the different subcarriers or of the different relays. More advanced techniques such as adaptive subcarrier allocation are required [10], [11].

Moreover, because relay-assisted OFDM transmissions are subjected to two-hop fading channels, dynamically rearranging the subcarriers at multiple relays may offer some additional diversity gains by appropriately pairing the subcarriers of the two hops. This subcarrier pairing philosophy was employed in [12], [13] for both amplify-and-forward (AF) and decodeand-forward (DF) relay-assisted single-user (SU) OFDM systems. This approach may also be applied in MU scenarios, such as in OFDMA, where the subcarriers of the two hops may be paired on a per-user basis, hence minimizing the multiuser interference (MUI). In addition, the corresponding resource allocation and MU scheduling may be carried out on a subbandgroup basis per user in the frequency domain (FD).

As a further advance, multiple relay selection schemes were investigated in [14]-[16] in an SU scenario when the CSI is available to the receivers but not to the transmitters. In this case, selecting multiple relays can increase the achievable cooperative/spatial diversity gain, but as a result, the potential selection diversity gain will be decreased when the total number of ORs is the same. Meanwhile, the interference among the multiple activated relays should be mitigated, which imposes further complexity on the transceivers. By contrast, when the CSI is available to both the receivers and the transmitters, optimum transmit beamforming was implemented through multiple 
relays in [17] and [18]. In that case, the signals from the relays coherently add at the receivers. Naturally, the elimination of the interference would require sophisticated algorithms, hence increasing the complexity imposed.

Therefore, carefully assigning the subbands/subcarriers in the context of OR can improve the diversity gain by avoiding the extra multirelay interference while retaining the MUI-free nature of SC-FDMA and OFDMA systems.

Although both the dynamic relay selection (DRS) [19], [20] and dynamic subband/subcarrier allocation (DSA) techniques [21] can provide a power gain, conventional DSA-aided OR may not exploit both. The main reason is that the two hops of the relay channel limit the capacity to that of the lower capacity link of the two hops. In the context of OR-assisted SC-FDMA systems, both the AF and DF relays limit the attainable MU performance, depending on the quality of the first hop. The consideration of the received signal at multiple relays can further improve the performance of dynamic resource allocation (DRA)-aided OR systems.

As a further advantage of channel-coded OR, the length of the interleavers combined with forward error correction (FEC) may be shortened, because the effects of fading are further randomized by OR. As a benefit, the delay of the entire FECcoded DF-relaying aided system is reduced. To the best of our knowledge, these issues have not been considered in the open literature.

In this paper, we propose two DRA strategies that were designed for the OR-assisted SC-FDMA uplink, where the ORs may invoke the soft-decision aid DF protocol [22], [23]. We assume that the multiple relays can exchange the pilot-aided channel-quality information (CQI) of all the users, facilitating the cooperation at the relays to carry out joint dynamic resource allocation (JDRA). ${ }^{1}$ Moreover, our assumption is that multiple relays participate in the multiway relaying (MWR) procedure for CQI exchange, as shown in Fig. 1(b), rather than for data exchange in [24]. The main contributions of this paper can be summarized as follows.

- In contrast to the aforementioned solutions, our focus is mainly on the energy efficiency of the DRA-aided OR-assisted SC-FDMA system, where the energy efficiency is quantified in terms of the energy consumption gain (ECG) and the energy reduction gain (ERG) [20], [25]. In addition, we evaluated the impact of the channel estimation overhead and the MWR-aided CQI exchange overhead on the energy efficiency.

- We dedicate special attention to the "first-hop quality awareness" of our DRA schemes. In particular, we explore any potential extra selection diversity to reduce the transmit power required and/or to improve the attainable system performance. To this end, we combine DRS and DSA based on the associated first-hop transmission qualities. Meanwhile, two types of first-hop quality aware (FHQA) JDRA algorithms are investigated, depending on whether the source-to-relay ( $\mathrm{S}-\mathrm{R})$ or the relay-to-

\footnotetext{
${ }^{1}$ The original MUI exchange scheme that operates through a single relay was referred to as MWR [24] for describing the data exchange that takes place among multiple data sources and destinations.
}

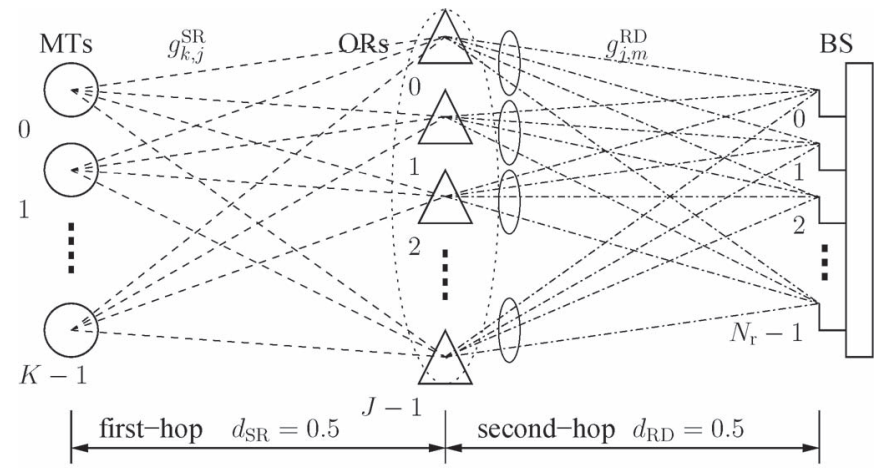

(a)

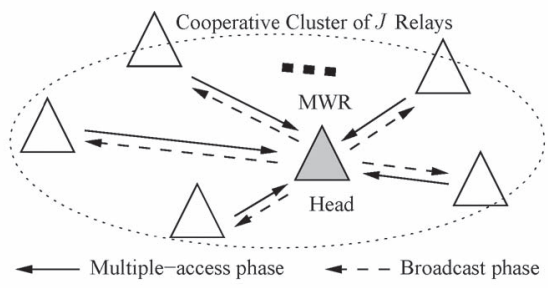

(b)

Fig. 1. OR-based MU SC-FDMA uplink through CQI exchange. (a) Opportunistically relayed SC-FDMA uplink. (b) Pilot-aided CQI exchange in a cooperative relay cluster by assuming MWR.

destination (R-D) channel quality dominates the attainable performance when the system invokes either single or multiple antennas at the base station (BS) receiver.

- Meanwhile, upon the "buffering delay awareness" of our interleaver-aided channel-coded systems, we quantify the benefits of OR combined with DSA in the context of interleaver-aided DF relaying for transmission over correlated fading channels in terms of reducing the interleaving delay and/or the total transmit power through joint OR and DSA.

- Our investigations involve the turbo equalization [26] in the FD, i.e., the minimum-mean-square-error (MMSE)criterion-based frequency-domain linear equalizer (FD-LE) using iterative joint equalization and decoding [26]. In addition, the performance benefits of both single-antenna and multiantenna-aided BS receivers have been considered for designing the bit-interleaved coded modulation (BICM) [27] aided SC-FDMA system.

This paper is organized as follows. In Section II, we will first outline the system model of the OR-assisted SC-FDMA uplink. In Sections III, we investigate the resource allocation schemes and outline the signal detection procedures employed at Multiantenna-aided BS receiver, respectively. The attainable performance of our proposed schemes is quantified by the simulation results in Section IV. Finally, we conclude in Section V.

\section{Opportunistic Relay-Assisted Single CARRIER-FREQUENCY DIVISION MULTIPLE ACCESS System Model}

\section{A. Scope and Assumptions}

Fig. 1(a) illustrates the topology of the OR-assisted system model. The OR-assisted SC-FDMA system considered 
(a)

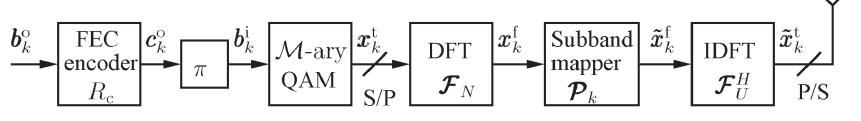

(b)

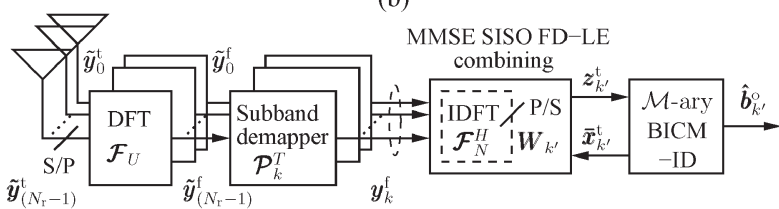

(c)

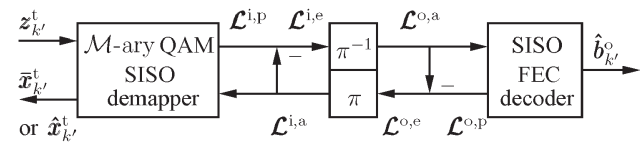

Fig. 2. Schematic of the BICM-aided SC-FDMA transceiver. (a) Transmitter schematic of the source MT and the DF relay. (b) Receiver schematic that invokes the MMSE turbo FD-LE of the DF relay and the BS. (c) Decoder schematic of the BICM-ID block in (b).

supports the $K$ uplink users, which forms a set $\mathcal{K}$, referred to as the source MTs of a traffic cell. The idle terminals located in each other's vicinity are members of the set $\mathcal{J}$, which may act as a cluster of relays. We assume that the relays of a cluster are located midway between the source MT and the destination BS. Hence, they are assumed to experience an identical pass loss. Each source MT, such as the $k$ th user's, is dynamically assigned to a single selected relay, whereas each relay can forward up to $K$ users' signals at a time so that the $j$ th relay may serve the specific users hosed by the paired set $\mathcal{K}_{j}$. We note that no relays are involved in the data transmissions when the set of eligible relays is empty, i.e., we have $\mathcal{K}_{j}=\varnothing$.

\section{B. Source MT's Transmitter Model}

1) Transmitted Signal of the Source MT: The channelcoded SC-FDMA transmitter adopted the BICM structure in Fig. 2(a). In particular, at the $k$ th source MT, the $N_{\mathrm{b}}$-length binary source data stream $\boldsymbol{b}_{k}^{\circ}$ is initially encoded by an outer channel code, such as a recursive systematic convolutional (RSC) code or a turbo code, with a coding rate of $R_{\mathrm{c}}$. Then, the outer encoded bit stream $c_{k}^{\circ}$ in Fig. 2(a) is interleaved by an $N_{\mathrm{c}}$-length random bitwise interleaver $\pi_{\mathrm{S}}$, where we have $N_{\mathrm{c}}=N_{\mathrm{b}} / R_{\mathrm{c}}$. The interleaver's output bit stream $\boldsymbol{b}_{k}^{\mathrm{i}}=$ $\left[\left(\boldsymbol{b}_{k, 0}^{\mathrm{i}}\right)^{T},\left(\boldsymbol{b}_{k, 1}^{\mathrm{i}}\right)^{T}, \ldots,\left(\boldsymbol{b}_{k,\left(N_{\mathrm{s}}-1\right)}^{\mathrm{i}}\right)^{T}\right]^{T}$ is fed into the $\mathcal{M}$-ary quadrature-amplitude modulation (QAM) mapper that transmits $Q$ b per symbol, where we have $N_{\mathrm{s}}=N_{\mathrm{c}} / Q$ and $\mathcal{M}=$ $2^{Q}$. Hence, we partition the $N_{\mathrm{c}}$-length bit sequence $\boldsymbol{b}_{k}^{\mathrm{i}}$ in Fig. 2(a) into $N_{\mathrm{s}}$ segments $\boldsymbol{b}_{k, n_{\mathrm{s}}}^{\mathrm{i}}$, each having a length of $Q$ for $n_{\mathrm{s}}=0,1, \ldots, N_{\mathrm{s}}-1$. Moreover, the $N_{\mathrm{s}}$-length modulated symbol sequence of Fig. 2(a) is then converted to $N_{\mathrm{v}}$ symbol vectors $\boldsymbol{x}_{k}^{\mathrm{S}, \mathrm{t}}\left[n_{\mathrm{v}}\right],\left(\forall n_{\mathrm{v}}=0,1, \ldots, N_{\mathrm{v}}-1\right)$, in which each vector $\boldsymbol{x}_{k}^{\mathrm{S}, \mathrm{t}}$ contains $N$ consecutive modulated symbol elements $x_{k, n}^{\mathrm{S}, \mathrm{t}}$ in the TD, where we have $N_{\mathrm{v}}=N_{\mathrm{s}} / N$ for $n=0$, $1, \ldots, N-1$. We refer to the symbol vector $x_{k}^{\mathrm{S}, \mathrm{t}}$ as an $S C$ FDMA symbol.

Furthermore, as shown in Fig. 2(a), the symbol vector is transformed by an $N$-point discrete Fourier transform (DFT) [28] from the TD to the FD, yielding $\boldsymbol{x}_{k}^{\mathrm{S}, \mathrm{f}}=\mathcal{F}_{N} \boldsymbol{x}_{k}^{\mathrm{S}, \mathrm{t}}$, where
$\mathcal{F}_{N}$ denotes the normalized $N$-point fast Fourier transform (FFT) matrix. The $k$ th user's resulting symbols are then mapped to the most appropriate $N$ subbands selected from the entire set of $U=(M \times N)$ subbands through the matrix $\mathcal{P}_{k}^{\mathrm{S}}$ at the source MT, which is referred to as subband mapping, where $M$ represents the bandwidth expansion factor. Following the subband mapping operations, the resulting $U$-element FD symbol vector is transformed to the TD by the $U$-point inverse discrete Fourier transform (IDFT), which is similar to the action of the OFDM transmitter [29]. Consequently, the $U$-symbol baseband-equivalent discrete-time signal $s_{k}^{\mathrm{S}, \mathrm{t}}$ transmitted by the $k$ th source MT before inserting the cyclic prefix $(\mathrm{CP})$ may be expressed as [20]

$$
\boldsymbol{s}_{k}^{\mathrm{S}, \mathrm{t}}=\sqrt{P_{k}^{\mathrm{S}}} \mathcal{F}_{U}^{H} \mathcal{P}_{k}^{\mathrm{S}} \mathcal{F}_{N} \boldsymbol{x}_{k}^{\mathrm{S}, \mathrm{t}}
$$

where the superscript ${ }^{t}$ refers to the TD signal. We assume that the source and relay equally share the unity transmit power, i.e., we have $P_{k}^{\mathrm{S}}=P_{k_{\tilde{j}}}^{\mathrm{R}}=0.5$, whereas $\mathcal{F}_{U}^{H}$ denotes the normalized $U$-point inverse fast Fourier transform (IFFT) matrix [28].

2) Localized Subband Mapping That Invokes DSA: The subband mapping regime guarantees that the maximum number of orthogonal users that may be supported for transmissions in a single time slot is equal to the bandwidth expansion factor; hence, we have $K \leqslant M$. Furthermore, the localized frequencydivision multiple access (LFDMA) is employed, which allows each user's SC-FDMA symbols to be mapped to $N$ consecutive subbands in the entire set of $U$ subbands. ${ }^{2}$ In particular, in our system, the subbands are allocated according to the LFDMA philosophy as

$$
\mathcal{P}_{u, n}^{(k, m)}= \begin{cases}1, & \text { if } u=m N+n \\ 0, & \text { otherwise }\end{cases}
$$

for $m=0,1, \ldots, M-1, n=0,1, \ldots, N-1$, and $u=0,1, \ldots$, $U-1$, where $\mathcal{P}_{u, n}^{(k, m)}$ is the $(u, n)$ th entry of $\mathcal{P}_{k}^{\mathrm{S}}$, and $m \in \mathcal{M}$ refers to the index of the individual spectral blocks. Each block consists of $N$ consecutive subbands in the so-called subband group defined for resource allocation. In addition, we define the static subband allocation (SSA) regime as having $m=k$ in (2), which is, in fact, the conventional LFDMA. By contrast, $m \neq k$ is referred to as the DSA, which is detailed in Section III.

\section{Relaying Models}

1) Signal Reception at the Relay: Assume that there are $J$ relay candidates, all of which can listen to all the $K$ source MTs. Then, after the classic CP removal, the TD signal that is received at the $j$ th relay may be expressed as

$$
\boldsymbol{r}_{j}^{\mathrm{R}, \mathrm{t}}=\sqrt{G_{\mathrm{SR}}} \sum_{k \in \mathcal{K}} \tilde{\boldsymbol{H}}_{k, j}^{\mathrm{SR}, \mathrm{t}} \boldsymbol{s}_{k}^{\mathrm{S}, \mathrm{t}}+\tilde{\boldsymbol{n}}_{j}^{\mathrm{R}, \mathrm{t}}
$$

\footnotetext{
${ }^{2}$ Another typical subband mapping scheme is referred to as the interleaved frequency-division multiple access (IFDMA), in which each user's symbols are evenly distributed over the entire system bandwidth. Although IFDMA may also invoke DSA, the achievable selection diversity gain may be lower than that of LFDMA operating through DSA, which is a consequence of the frequency selectivity of the IFDMA symbol spectrum.
} 
where $\tilde{\boldsymbol{H}}_{k, j}^{\mathrm{SR}, \mathrm{t}}$ hosts the $(U \times U)$-element TD channel coefficient matrix of the S-R links from the $k$ th source $\mathrm{MT}$ to the $j$ th relay, whereas $\tilde{\boldsymbol{n}}_{j}^{\mathrm{R}, \mathrm{t}}$ represents the $U$-element complex-valued Additive white Gaussian noise (AWGN) vectors with zero mean and a variance of $\sigma_{N}^{2}$ with regard to each element.

The signals $\boldsymbol{r}_{j}^{\mathrm{R}, \mathrm{t}}$ received by the $j$ th relay is first transformed to the FD by the $U$-point DFT operation and then demapped to the appropriate $N$ subbands through the matrix $\left(\mathcal{P}_{k_{j}}^{\mathrm{S}}\right)^{T}$ for user $k_{j}$ as defined in (2), yielding its equivalent FD representation in the form of [20]

$$
\boldsymbol{y}_{k_{j}}^{\mathrm{R}, \mathrm{f}}=\sqrt{G_{\mathrm{SR}}} \boldsymbol{H}_{k, j}^{\mathrm{SR}, \mathrm{f}} \boldsymbol{x}_{k, j}^{\mathrm{S}, \mathrm{f}}+\left(\mathcal{P}_{k_{j}}^{\mathrm{S}}\right)^{T} \mathcal{F}_{U} \tilde{\boldsymbol{n}}_{j}^{\mathrm{R}, \mathrm{t}}
$$

where $\boldsymbol{H}_{k, j}^{\mathrm{SR}, \mathrm{f}}$ is the $(N \times N)$-element equivalent FD channel coefficient matrix after subband demapping, which is given by

$$
\begin{aligned}
\boldsymbol{H}_{k, j}^{\mathrm{SR}, \mathrm{f}} & =\left(\mathcal{P}_{k_{j}}^{\mathrm{S}}\right)^{T} \mathcal{F}_{U} \tilde{\boldsymbol{H}}_{k, j}^{\mathrm{SR}, \mathrm{t}} \mathcal{F}_{U}^{H} \mathcal{P}_{k_{j}}^{\mathrm{S}} \\
& =\operatorname{diag}\left[h_{k, j, 0}^{\mathrm{SR}, \mathrm{f}}, h_{k, j, 1}^{\mathrm{SR}, \mathrm{f}}, \ldots, h_{k, j,(N-1)}^{\mathrm{SR}, \mathrm{f}}\right] .
\end{aligned}
$$

2) Transmitted Signal of the Relays: We assume that the transmitter of the SC-FDMA relay is identical to that of the source MT. Before inserting the $\mathrm{CP}$ at the $j$ th relay of the set $\mathcal{J}$, the $U$-element TD transmitted signal $s_{j}^{\mathrm{R}, \mathrm{t}}$ that contains the $k_{\breve{j}} \in \mathcal{K}_{\breve{j}}$ source users' data may be expressed as

$$
\boldsymbol{s}_{j}^{\mathrm{R}, \mathrm{t}}=\sum_{k \in \mathcal{K}} \rho_{k}, \boldsymbol{s}_{k, j}^{\mathrm{R}, \mathrm{t}}=\sum_{k_{\tilde{j}} \in \mathcal{K}_{\tilde{j}}} \boldsymbol{s}_{k_{\tilde{j}}}^{\mathrm{R}, \mathrm{t}}
$$

where $\rho_{k, j}$ is the S-R pairing factor of the $k$ th source and the $j$ th relay defined by $\rho_{k, j}=1$ if $k=k_{j}$ and $j=\check{j}$; otherwise, we have $\rho_{k, j}=0$. As shown in (6), based on the set of $J$ relays, only the relays $\check{j}$ that were selected for the set $\breve{\mathcal{J}}$ forward the source data of user $k_{\tilde{j}}$ in the set $\mathcal{K}_{\tilde{j}}$, whereas the remaining candidate relays are not allowed to transmit.

3) Soft-DF Protocol: The receiver of the DF relay invokes the MMSE-based turbo FD-LE, as detailed in Section II-D2. As shown in Fig. 2(b), the $k_{j}$ th user's first-hop FD received signal vectors $\boldsymbol{y}_{k_{j}}^{\mathrm{R}, \mathrm{f}}\left[n_{\mathrm{v}}\right]$ at the $j$ th relay, for $\left(n_{\mathrm{v}}=0,1, \ldots\right.$, $\left.N_{\mathrm{v}}-1\right)$ and $\left(N_{\mathrm{v}}=N_{\mathrm{s}} / N\right)$, are equalized by the a posteriori probability-based MMSE FD-LE combined with the symbolto-bits demapper. All signals are represented in terms of their logarithmic likelihood ratio (LLR), as shown in the iterative structure in Fig. 2(c), which is based on the concept of BICM using iterative decoding (BICM-ID) and detailed in [27].

To reduce the detrimental effects of error propagation, soft decisions may be invoked [22], [23]. In particular, instead of using extrinsic LLRs $\mathcal{L}^{\mathrm{o}, \mathrm{e}}\left(\boldsymbol{b}_{k_{\dot{j}}}^{\mathrm{o}}\right)$, the extrinsic LLRs related to the encoded bit stream $\boldsymbol{c}_{k_{\tilde{j}}}^{\mathrm{o}}$ in Fig. 2(a) can be used as the relevant soft-decision variables. After appropriate reordering of the coded bits in the sequence by the soft-bit interleaver $\pi_{R}$ in Fig. 2(c), the resulting LLRs $\mathcal{L}^{\mathrm{o}, \mathrm{e}}\left(\boldsymbol{c}_{k_{\tilde{j}}}^{\mathrm{o}}\right)$ become the a priori information $\mathcal{L}^{\mathrm{i}, \mathrm{a}}\left(\boldsymbol{b}_{k_{\tilde{r}}}^{\mathrm{i}}\right)$ in Fig. 2(c), which allows us to calculate the bit probability $P\left(b_{k_{\tilde{j}}, n_{\mathrm{s}}, q}=\mathrm{b}_{i, q}\right)$, for $q=0,1, \ldots, Q-1$.
Then, the symbol probability of the transmitted symbols of the $k_{j}$ th source MT may be further expressed by [26]

$$
P\left(x_{k_{j}, n_{\mathrm{s}}}^{\mathrm{S}, \mathrm{t}}=\mathrm{s}_{i}\right)=\prod_{q=0}^{Q-1} \frac{1}{2}\left(1+\stackrel{\circ}{\mathrm{b}}_{i, q} \tanh \frac{\mathcal{L}_{k_{j}}^{\mathrm{i}, \mathrm{a}},\left(n_{\mathrm{s}} Q+q\right)}{2}\right)
$$

where $n_{\mathrm{s}}=n_{\mathrm{v}} N+n$ for $n_{\mathrm{s}}=0,1, \ldots,\left(N_{\mathrm{s}}-1\right), n=0,1, \ldots$, $(N-1)$, and $q=0,1, \ldots,(Q-1)$. Let the symbols of the $k$ th user forwarded by the $\check{j}$ th DF relay be expressed as $\bar{x}_{k_{\tilde{j}}, n_{\mathrm{s}}}^{\mathrm{S}, \mathrm{t}}$, which are expressed in terms of the expectation values of the transmitted symbols of $x_{k_{\tilde{j}}, n_{\mathrm{s}}}^{\mathrm{S}, \mathrm{t}}$ as [26]

$$
x_{k_{\tilde{j}}, n_{\mathrm{s}}}^{\mathrm{R}, \mathrm{t}}=\bar{x}_{k_{\tilde{j}}, n_{\mathrm{s}}}^{\mathrm{S} \mathrm{t}}=\sum_{\mathrm{s}_{i} \in \mathcal{S}} \mathrm{s}_{i} \cdot P\left(x_{k_{\tilde{j}}, n_{\mathrm{s}}}^{\mathrm{S}, \mathrm{t}}=\mathrm{s}_{i}\right) .
$$

Finally, the $n_{\mathrm{v}}$ th $N$-element DF symbol vector $x_{k_{j}}^{\mathrm{R}, \mathrm{t}}\left[n_{\mathrm{v}}\right]=$ $\left[x_{k_{\tilde{j}}, 1}^{\mathrm{R}, \mathrm{t}}, x_{k_{\tilde{j}}, 1}^{\mathrm{R}, \mathrm{t}}, \ldots, x_{k_{\tilde{j}},(N-1)}^{\mathrm{R}, \mathrm{t}}\right]^{T}$ is used for generating the $k$ th user's $U$-element transmitted symbol $s_{k_{\tilde{j}}}^{\mathrm{R}, \mathrm{t}}$ at the $\check{j}$ th relay, yielding

$$
\boldsymbol{s}_{k_{\tilde{j}}}^{\mathrm{R}, \mathrm{t}}=\sqrt{P_{k_{\tilde{j}}}^{\mathrm{R}}} \mathcal{F}_{U}^{H} \mathcal{P}_{k_{\tilde{j}}}^{\mathrm{R}} \mathcal{F}_{N} \boldsymbol{x}_{k_{\tilde{j}}}^{\mathrm{R}, \mathrm{t}}
$$

without attaching the CP. In (9), the subband allocation of the corresponding S-R pair is carried out by the matrix $\mathcal{P}_{k_{\tilde{j}}}^{\mathrm{R}}$.

\section{Multiantenna-Aided BS Receiver Model}

1) DF Signal Received at the BS: Because we assume that the BS's receiver invokes $N_{\mathrm{r}}$ antennas, the $U$-element TD signal vector that holds the $K$ user's data received at the $n_{\mathrm{r}}$ th BS antenna from the selected relays in the set $\check{\mathcal{J}}$ may be expressed as

$$
\tilde{\boldsymbol{y}}_{n_{\mathrm{r}}}^{\mathrm{D}, \mathrm{t}}=\sum_{\tilde{j} \in \tilde{\mathcal{J}}} \sqrt{G_{\mathrm{RD}}} \tilde{\boldsymbol{H}}_{\tilde{j}, n_{\mathrm{r}}}^{\mathrm{RD}, \mathrm{t}} \sum_{k_{\tilde{j}} \in \mathcal{K}_{\tilde{j}}} \boldsymbol{s}_{k_{\tilde{j}}}^{\mathrm{R}, \mathrm{t}}+\tilde{\boldsymbol{n}}_{n_{\mathrm{r}}}^{\mathrm{D}, \mathrm{t}}
$$

where $\tilde{\boldsymbol{H}}_{\tilde{j}, n_{\mathrm{r}}}^{\mathrm{RD} \text { d }}$ denotes the $(U \times U)$-element TD circulant channel coefficient matrix between relay $\breve{j}$ and the $n_{\mathrm{r}}$ th antenna. Next, the $U$-point FFT carried out at each antenna transforms the TD signal $\tilde{\boldsymbol{y}}_{n_{\mathrm{r}}}^{\mathrm{D}, \mathrm{t}}$ into the FD, where the signal of user $k_{\tilde{j}}$ is then demapped into an appropriate subband group according to the subband allocation matrix by $\mathcal{P}_{k_{\tilde{j}}}^{\mathrm{R}}$ at the relay. In the DF scenario, the BS's receiver only detects the signal transmitted by the relay, which relies only on the CSI of the R-D link. Hence, we have the FD received signal of user $k_{\breve{j}}$ at the $n_{\mathrm{r}}$ th antenna as

$$
\boldsymbol{y}_{n_{\mathrm{r}}, k_{\tilde{j}}}^{\mathrm{D}, \mathrm{f}}=\sqrt{P_{k_{\tilde{j}}}^{\mathrm{R}} G_{\mathrm{RD}}} \boldsymbol{H}_{k_{\tilde{j}}, n_{\mathrm{r}}}^{\mathrm{RD}, \mathrm{f}} \mathcal{F}_{N} \boldsymbol{x}_{k}^{\mathrm{R}, \mathrm{t}}+\boldsymbol{n}_{n_{\mathrm{r}}, k_{\tilde{j}}}^{\mathrm{D}, \mathrm{f}}
$$

where $\boldsymbol{H}_{k_{\tilde{j}}, n_{\mathrm{r}}}^{\mathrm{RD}, \mathrm{f}}$ denotes the equivalent FD channel matrix for reception by the $n_{\mathrm{r}}$ th antenna of the R-D link, whereas the noise contribution $\boldsymbol{n}_{n_{\mathrm{r}}, k_{\tilde{j}}}^{\mathrm{D}, \mathrm{f}}=\left(\mathcal{P}_{k_{\tilde{j}}}^{\mathrm{R}}\right)^{T} \mathcal{F}_{U} \tilde{\boldsymbol{n}}_{n_{\mathrm{r}}, k_{\tilde{j}}}^{\mathrm{D}, \mathrm{t}}$ is imposed at the $n_{\mathrm{r}}$ th antenna, and the corresponding power of the $n$th element is $\mathcal{N}_{n_{r}, n}=\sigma_{\mathrm{N}}^{2}$. In addition, for receiver diversity combining, we arrive at the $N_{\mathrm{r}} N$-element power-normalized FD 
received signal of user $k^{\prime}=k_{\breve{j}}$ through $N_{\mathrm{r}}$ antennas, which is formulated as

$$
\boldsymbol{y}_{k^{\prime}}^{\mathrm{f}}=\boldsymbol{H}_{k^{\prime}} \boldsymbol{x}_{k^{\prime}}^{\mathrm{R}, \mathrm{t}}+\boldsymbol{n}_{k^{\prime}}^{\mathrm{f}}
$$

where we have

$$
\begin{gathered}
\boldsymbol{y}_{k^{\prime}}^{\mathrm{f}}=\sqrt{1 / P_{k_{\tilde{j}}}^{\mathrm{R}}}\left[\left(\boldsymbol{y}_{0, k_{\tilde{j}}}^{\mathrm{D}, \mathrm{f}}\right)^{T},\left(\boldsymbol{y}_{1, k_{\tilde{j}}}^{\mathrm{D}, \mathrm{f}}\right)^{T}, \ldots,\left(\boldsymbol{y}_{\left(N_{\mathrm{r}}-1\right), k_{\tilde{j}}}^{\mathrm{D}, \mathrm{f}}\right)^{T}\right]^{T} \\
\boldsymbol{H}_{k^{\prime}=} \boldsymbol{H}_{k^{\prime}}^{\mathrm{f}} \mathcal{F}_{N} \\
\boldsymbol{H}_{k^{\prime}}^{\mathrm{f}}=\sqrt{G_{\mathrm{RD}}}\left[\left(\boldsymbol{H}_{k_{\tilde{j}}, \mathrm{f}}^{\mathrm{RD}, \mathrm{f}}\right)^{T},\left(\boldsymbol{H}_{k_{\tilde{j}}, 1}^{\mathrm{RD}, \mathrm{f}}\right)^{T}\right. \\
\left.\ldots,\left(\boldsymbol{H}_{k_{\tilde{j}},\left(N_{\mathrm{r}}-1\right)}^{\mathrm{RD}, \mathrm{f}}\right)^{T}\right]^{T} \\
\boldsymbol{n}_{k^{\prime}}^{\mathrm{f}}=\sqrt{1 / P_{k_{\tilde{j}}}^{\mathrm{R}}}\left[\left(\boldsymbol{n}_{0, k_{\tilde{j}}}^{\mathrm{D}, \mathrm{f}}\right)^{T},\left(\boldsymbol{n}_{1, k_{\tilde{j}}}^{\mathrm{D}, \mathrm{f}}\right)^{T}, \ldots,\left(\boldsymbol{n}_{\left(N_{\mathrm{r}}-1\right), k_{\tilde{j}}}^{\mathrm{D}, \mathrm{f}}\right)^{T}\right]^{T} .
\end{gathered}
$$

In particular, $\boldsymbol{H}_{k^{\prime}}$ denotes the $\left(N_{\mathrm{r}} N \times N\right)$-element equivalent interference channel coefficient matrix that invokes $N$-point DFT spreading ${ }^{3}$ of user $k^{\prime}$, where $\boldsymbol{n}_{k^{\prime}}^{\mathrm{f}}$ holds the normalized noise imposed on the $N_{\mathrm{r}}$ antennas.

2) FD Turbo Equalization: The BS's receiver that is considered for channel-coded SC-FDMA systems may be designed by invoking the complex-valued MMSE turbo FD-LE in Fig. 2(b) based on the BICM-ID scheme in Fig. 2(c), where the corresponding iterative joint equalization and decoding philosophy was discussed in Section II-C3. Following the initial filtering by the classic MMSE FD-LE and BICM decoding, these blocks exchange extrinsic information in a number of consecutive iterations [26]. To further elaborate a little, the mean of $\boldsymbol{x}_{k^{\prime}}^{\mathrm{t}}$, denoted by $\boldsymbol{x}_{k^{\prime}}^{\mathrm{t}}$ in Fig. 2(b), is generated by the bit-probabilityto-symbol-probability conversion using a priori LLRs, which are generated by appropriately ordering them by the interleaver based on the feedback of the BICM decoder in Fig. 2(c). Therefore, by applying soft-information exchange between the equalizer and the decoder, the MMSE weight matrix is updated based on the a priori information gleaned during each iteration. This regime further reduces the residual ISI that is imposed on the estimated symbols. The design philosophy of the MMSE turbo FD-LE receiver was detailed in [26], [30], and [31].

\section{DRA FOR OR}

In the OR channels that we considered, both the spatial- and spectral-domain resources offered by multiple relays may be explored for power reduction. To achieve a selection diversity gain, the DRS allows each user to benefit from exploring $J$ different S-R channels and $J$ R-D channels. The corresponding complex-valued fading envelope may be deemed to be independent and identically distributed (i.i.d.) for each of these links of the resulting virtual MIMO scheme. Furthermore, the DSA beneficially rearranges the MU's signals for transmission

\footnotetext{
${ }^{3}$ As a result, the corresponding equalizer weight matrix $\boldsymbol{W}_{k^{\prime}}$ in Fig. 2(b) also invokes the $N$-point DFT operation $\mathcal{F}_{N}$. Therefore, the $N$-point IDFT operation at the receiver is not necessary after applying $\boldsymbol{W}_{k^{\prime}}^{H}$, as evidenced by the dashed block in Fig. 2(b).
}

over the most appropriate subband groups for the second-hop relaying. In addition, experiencing frequency-selective fading in the DRS-assisted OR scheme may provide an additional MU diversity gain for the system. Therefore, the beneficial combination of DRS and DSA can assign appropriate desired subband groups for conveying the MUs' signals regenerated at the appropriate relays, each of which may dynamically serve MUs.

FHQA JDRA: Although the conventional combination of DRS and DSA achieves a diversity gain through beneficial subband allocation and relay selection, the degree of freedom that is associated with beneficially allocating MU's signals across the entire set of $(M \times J)$ subband groups of the $J$ relays has not been fully exploited. Unless near-error-free decoding at the DF relays is possible, the MU's signals that were received and forwarded by the DF relays may result in error propagation at the BS due to the first-hop transmissions in terms of the S-R CQI. This phenomenon motivates us to design and investigate the first-hop quality-aware (FHQA) JDRA schemes conceived for OR-assisted SC-FDMA. We assume that the CSIs of the $K$ S-R links and the $\left(M \times J \times N_{\mathrm{r}}\right)$ R-D links are perfectly estimated at each relay's receiver. We also assume that these cooperating relays can exchange their CQIs. More specifically, as mentioned in Section I, the $(J-1)$ relays in Fig. 1(b) [24] transmit their CQI to an appropriately selected relay that acts as a cluster head, which then broadcasts the CQI back to the $(J-1)$ relays. The relevant decisions are made at the relay rather than at the BS. This implies that the BS's receiver does not require the CQI of the first hop.

In this section, the following three schemes are investigated: 1) conventional combination of DRS and DSA, denoted as DRS-DSA; 2) FHQA JDRA approach-1, denoted as JDRA-1; and 3) the FHQA JDRA approach-2, denoted as JDRA-2. The DRS-DSA scheme does not require any cooperation among the localized relays; hence, it is controlled by the BS, depending on the R-D CQI. By contrast, both the FHQA JDRA schemes rely on cooperation among the localized relays; hence, it is controlled by the relay cluster head. The flowcharts of FHQA JDRA schemes are portrayed in Fig. 3. Observe the differences in the procedures in Fig. 3(b) with respect to the DSA-DRS in Section III-C and the JDRA-1 scheme in Section III-D. The main differences between the two JDRA approaches may further be characterized by contrasting Fig. 3(b) with Fig. 3(a).

\section{A. CQI}

There are several reasons for grouping the $N$ subbands of each user together for relaying. The first reason is related to the relevant complexity consideration, because searching for the optimal solution on a group-by-group rather than a subband-bysubband basis is significantly less complex. Another reason for grouping the $N$ subbands of each user together is because each user's TD symbol generated after first-hop channel equalization at the relay is related to all the $N$ elements in $\boldsymbol{y}_{k_{j}}^{\mathrm{R}, \mathrm{f}}$ due to the well-understood nature of the IDFT employed at the SC-FDMA receiver. Therefore, we use the average $S-R$ channel attenuation of the $N$ subbands of the $k$ th user's signal received by the 


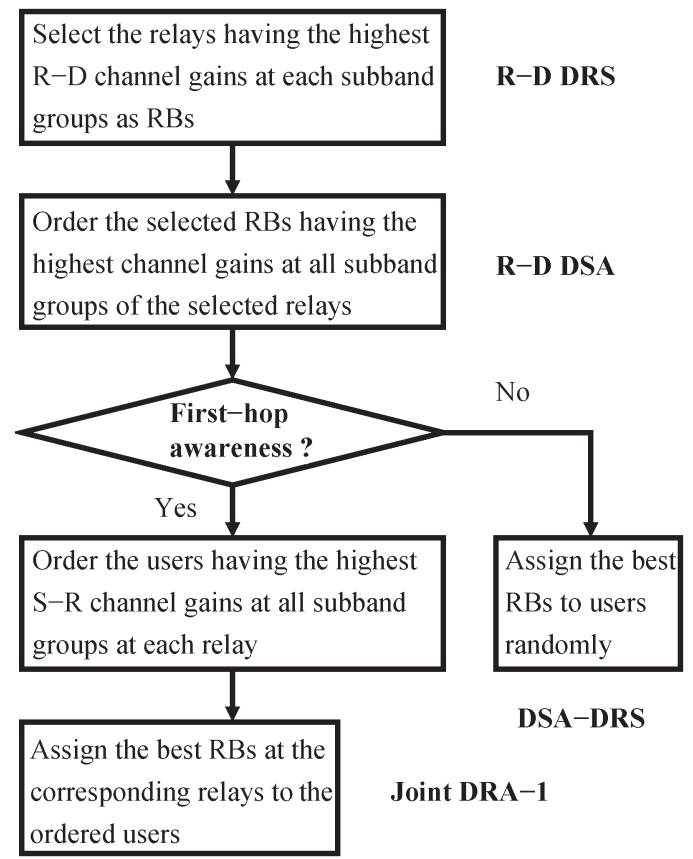

(a)

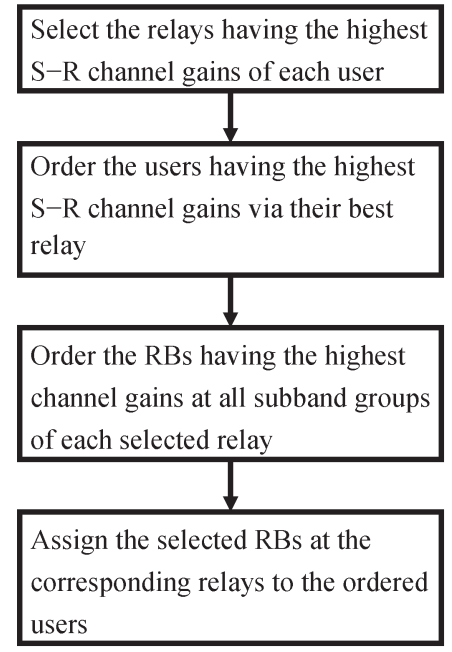

S-R DRS

First-hop

awareness

R-D DSA

\section{Joint DRA-2}

(b)

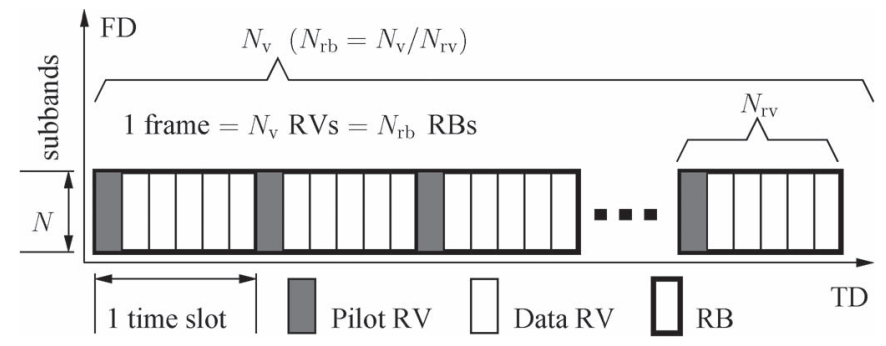

Fig. 4. Resource allocation frame structure in the SC-FDMA uplink scheme in Fig. 1(a).

\section{B. Relaying Frame Structure}

Observe in Fig. 4 that, in our DRA-aided OR system, each SC-FDMA symbol in the FD is referred to as a resource vector $(\mathrm{RV})$, where the components of the $\mathrm{RV}$ are the original symbols that modulate each of the subbands. Furthermore, $N_{\mathrm{rv}}$ consecutive RVs in the TD constitute a resource block (RB) in the uplink during a single time slot, which constitutes the basic unit of resource allocation. The first RV may be regarded as the reference, i.e., the pilot RV in Fig. 4, which is known to both the transmitter and the receiver and, hence, may be used for channel estimation. Then, both the subband allocation and the relay selection valid for the corresponding time slot are particularly optimized for the channel quality that was estimated through this pilot RV. Hence, we have a total of $N_{\mathrm{rb}}=N_{\mathrm{v}} / N_{\mathrm{rv}} \mathrm{RBs}$ within a frame of $N_{\mathrm{S}}$ QAM symbols. In particular, the number of RVs $N_{\mathrm{rv}}$ per RB determines the transmission overhead required, including the synchronization sequences, the pilot RVs invoked for the estimation of the S-R and R-D links, and the duration of the CQI exchange in Fig. 1(b) for the FHQA JDRA schemes. ${ }^{4}$ Naturally, increasing the overhead provides more accurate channel estimation in support of the CQI exchange; hence, it may result in increased energy efficiency, although it may reduce the system's throughput. The $N_{\text {rb }}$ RBs of each user are allocated by conventional static localized subband mapping during the first-hop transmissions, which reduces the effective throughput of the source MTs. However, during the second hop, each RB or, possibly, several consecutive RBs of a specific user may be rearranged at the appropriate relays, depending on the specific resource allocation schemes employed. Moreover, in the channel-coded system, the signal is interleaved and decoded on a frame-by-frame basis. Hence, an interleaver-depthdependent buffering delay is introduced when using iterative decoding at the DF relay. In addition, the specific number of consecutive RBs scheduled for concomitant transmission is typically determined by the normalized Doppler frequency $f_{\text {nd }}$, which is usually carried out without changing the subband group and relay.

We also assume that the BS invokes $N_{\mathrm{r}}$ receiver antennas, where the sum of channel attenuation averaged over multiple antennas may be considered as a relevant quality metric for the overall channel effects. The average attenuation of the $m$ th group of $N$ subbands in the channel spanning from the $j$ th relay to the BS is given by

$$
g_{j, m}^{\mathrm{RD}}=\frac{1}{N} \sum_{u=m N}^{m N+N-1} \sum_{n_{\mathrm{r}}=0}^{N_{\mathrm{r}}-1}\left|h_{j, u, n_{\mathrm{r}}}^{\mathrm{RD}, \mathrm{f}}\right|^{2} .
$$

${ }^{4}$ For simplicity, we assume that the transmissions of two-hop relaying and the CQI exchange are perfectly synchronized; hence, the practical synchronization issue is ignored. However, we evaluate the energy consumption expended in the relatively short duration of the pilots-based CQI exchange compared with the two-hop relaying. 
TABLE I

EXAMPlE of FHQA JDRA Schemes FOR $K=3, M=4$ AND $J=5$. (a) APPROACH-1 (JDRA-1). (b) ApPROACH-2 (JDRA-2)

\begin{tabular}{|c|c|c|c|c|c|c|c|}
\hline & \multicolumn{3}{|c|}{ First-hop $g_{k, j}^{\mathrm{SR}}$} & \multicolumn{4}{|c|}{ Second-hop $g_{j, m}^{\mathrm{RD}}$} \\
\hline & $k=0$ & $k=1$ & $k=2$ & $m=0$ & $m=1$ & $m=2$ & $m=3$ \\
\hline \multicolumn{8}{|c|}{ (a) Approach-1 (JDRA-1) } \\
\hline $\bar{j}=0$ & $0.05^{(3 r d)}$ & $0.53^{(2 n d)}$ & $2.26^{(1 s t)}$ & 0.04 & 0.33 & $\underline{1.90}^{(3 r d)}$ & 0.56 \\
\hline$j=1$ & $0.16^{(3 r d)}$ & $0.20^{(2 n d)}$ & $0.5 \mathbf{7}^{(1 s t)}$ & 0.70 & 1.91 & 0.84 & $\underline{4.41}^{(1 s t)}$ \\
\hline$j=2$ & 0.90 & 3.07 & 0.70 & 0.45 & 0.26 & 0.28 & 0.90 \\
\hline$j=3$ & $0.18^{(2 n d)}$ & $0.09^{(3 r d)}$ & $0.21^{(1 s t)}$ & $1.75^{(4 t h)}$ & $2.50^{(2 n d)}$ & 0.02 & 0.09 \\
\hline$j=4$ & 1.42 & 1.44 & 0.81 & 0.29 & 1.36 & 0.17 & 0.05 \\
\hline Target $(\check{j}, \check{m})$ & $(3,1)$ & $(0,2)$ & $(1,3)$ & & & & \\
\hline \multicolumn{8}{|c|}{ (b) Approach-2 (JDRA-2) } \\
\hline$\overline{j=0}$ & 0.05 & 0.53 & ${\underline{2.26^{(2 n d)}}}^{(2 n)}$ & $0.04^{(4 t h)}$ & $0.33^{(3 r d)}$ & $\underline{1.90}^{(1 s t)}$ & $0.56^{(2 n d)}$ \\
\hline$j=1$ & 0.16 & 0.20 & 0.57 & 0.70 & 1.91 & 0.84 & 4.41 \\
\hline$j=2$ & 0.90 & $\underline{\mathbf{3 . 0 7}}^{(1 s t)}$ & 0.70 & $0.45^{(2 n d)}$ & $0.26^{(4 t h)}$ & $0.28^{(3 r d)}$ & $0.90^{(1 s t)}$ \\
\hline$j=3$ & 0.18 & 0.09 & 0.21 & 1.75 & 2.50 & 0.02 & 0.09 \\
\hline$j=4$ & $\underline{\mathbf{1 . 4 2}}^{(3 r d)}$ & 1.44 & 0.81 & $0.29^{(2 n d)}$ & $\underline{\mathbf{1 . 3 6}}^{(1 s t)}$ & $0.17^{(3 r d)}$ & $0.05^{(4 t h)}$ \\
\hline Target $(\check{j}, \check{m})$ & $(4,1)$ & $(2,3)$ & $(0,2)$ & & & & \\
\hline
\end{tabular}

\section{Conventional Approach of DRS Combined With DSA}

The DRS-DSA scheme allows the MUs' received signals to adaptively be transmitted in the most appropriate subband groups at dynamically selected relays by taking into account the $(M \times J)$ possible subband groups of the $J$ relays. We describe this procedure in more detail as follows. ${ }^{5}$

1) Initialization: Set $\check{\mathcal{J}}=\varnothing, \stackrel{\mathcal{M}}{=}=\varnothing$.

2) Second-hop DRS: At the $m$ th subband group, $\forall m \in \mathcal{M}$ :

a) Obtain $\check{j}_{m}=\arg \max _{j \in \mathcal{J}}\left\{g_{j, m}^{\mathrm{RD}}\right\}$.

b) Update $\check{\mathcal{J}}=\check{\mathcal{J}} \cup\left\{\check{j}_{m}\right\}$ and $\check{\mathcal{G}}^{\mathrm{RD}}=\check{\mathcal{G}}^{\mathrm{RD}} \cup\left\{g_{\tilde{j}, m}^{\mathrm{RD}}\right\}$.

3) Second-hop DSA: For $i=0,1, \ldots, M-1$ :

a) Obtain $\stackrel{\circ}{m}_{i}=\arg \max _{m \in \mathcal{M}}\left\{g_{\tilde{j}, m}^{\mathrm{RD}}\right\}$.

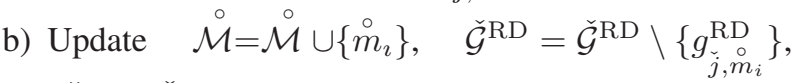
$\forall \check{j}_{m} \in \check{\mathcal{J}}$.

4) Fair user assignment: Randomly assign the user $\stackrel{\circ}{k}_{i}$ with the resource pair $\left(\stackrel{\circ}{m}_{i}, \breve{j}_{m_{i}}\right)$ for $i=0,1, \ldots, K-1$.

\section{FHQA Joint DRA Approach-1}

JDRA-1 optimizes the DRS-DSA scheme in Section III-C by allocating the RBs with the highest channel gain for the R-D links to the appropriate users, which are stored in an ordered user set at each relay in terms of their first-hop CQI. We assume that each relay stores and labels a sorted CQI set of the S-R links while receiving from $K$ users, and the resulting $J$ ordered CQI sets of the S-R links and the CQI sets of the R-D links are then exchanged among the $J$ relays through the relays' cluster

${ }^{5}$ Let us define the notations as follows. The notations $\mathcal{K}, \mathcal{J}, \mathcal{M}, \mathcal{G}^{\mathrm{SR}}$, and $\mathcal{G}^{\mathrm{RD}}$ denote the set of users $k=0,1,2, \ldots, K-1(\forall k \in \mathcal{K})$, the relays $j=$ $0,1,2, \ldots, J-1(\forall j \in \mathcal{J})$, the subband groups $m=0,1,2, \ldots, M-1$ $(\forall m \in \mathcal{M})$, the $\mathrm{S}-\mathrm{R}$ channel gains $\forall g_{k, j}^{\mathrm{SR}} \in \mathcal{G}^{\mathrm{SR}}$, and the R-D channel gains $\forall g_{j, m}^{\mathrm{RD}} \in \mathcal{G}^{\mathrm{RD}}$, respectively. Given the elements in the set $\mathcal{A},(\forall a \in \mathcal{A})$, the notation $\breve{a}$ represents the selected element of $a$ and is collected in the set $\breve{\mathcal{A}}$. The notation $\stackrel{\circ}{a}_{i}$ denotes the element $a$ with ordering index $i$ in the ordered version $\stackrel{\circ}{\mathcal{A}}$ of the original set $\mathcal{A}$. The notation $\varnothing$ represents the "clear" operation. The notation $\cup, \backslash$ indicates that the element in $\{a\}$ is incorporated or excluded from the set $\mathcal{A}$, respectively. head. The associated procedures may be described step by step as follows.

1) Second-hop DRS-DSA: Detailed in Section III-C.

2) FHQA Ordering: At the $j$ th relay, $\forall j \in \mathcal{J}$ :

a) $\operatorname{Set} \stackrel{\circ}{\mathcal{G}}_{j}^{\mathrm{SR}}=\varnothing, \stackrel{\circ}{\mathcal{K}}_{j}=\varnothing$.

b) Collect $\mathcal{G}_{j}^{\mathrm{SR}}=\left\{g_{0, j}^{\mathrm{SR}}, g_{1, j}^{\mathrm{SR}}, \ldots, g_{K-1, j}^{\mathrm{SR}}\right\}$.

c) Obtain the $i$ th-ordered user index in the set $\stackrel{\circ}{\mathcal{K}}_{j}: \stackrel{\circ}{k}_{i, j}=$ $\arg \max _{k \in \mathcal{K}}\left\{g_{k, j}^{\mathrm{SR}}\right\}$ for $i=0,1, \ldots, K-1$.

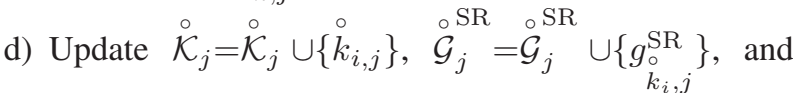
$\mathcal{G}_{j}^{\mathrm{SR}}=\mathcal{G}_{j}^{\mathrm{SR}} \backslash\left\{\underset{g_{i}, j}{\mathrm{SR}}\right\}$ for $i=0,1, \ldots, K-1$.

3) User assignment: Set $\stackrel{\circ}{\mathcal{K}}=\varnothing$; for the $\imath$ th user, $(\imath=$

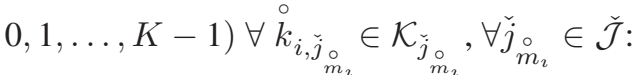

a) If ${\stackrel{\circ}{k_{i}, \check{j}_{\circ}}}_{m_{\imath}} \notin \stackrel{\circ}{\mathcal{K}}$, collect the user ${\stackrel{\circ}{k_{i, j_{\circ}}}}_{m_{\imath}}$ to the $\imath$ th element of $\stackrel{\circ}{\mathcal{K}}, \stackrel{\circ}{\mathcal{K}}=\stackrel{\circ}{\mathcal{K}} \cup\left\{{\stackrel{\circ}{k_{i, \check{j}_{\circ}}}}_{m_{\imath}}\right\}$. Update $\stackrel{\circ}{\mathcal{K}}_{\check{j}_{m_{\imath}}}=\stackrel{\circ}{\mathcal{K}}_{\check{j}_{\circ}} \backslash\left\{{\stackrel{\circ}{m_{\imath}}}_{i, \check{j}_{\circ}}\right\}$.

b) Otherwise, if $\stackrel{\circ}{k}_{i, \check{j}_{m_{2}}} \in \stackrel{\circ}{\mathcal{K}}$, we apply $i:=i+1$, return to step 3a.

Assign the user $\stackrel{\circ}{k}_{i, \check{j}_{m_{2}}}$ with the resource pair $\left(\stackrel{\circ}{m}_{i}, \check{j}_{\dot{m}_{i}}\right)$.

For example, Table I(a) characterizes the allocation strategy based on the aforementioned FHQA JDRA-1 procedure. Let us consider the $\mathrm{S}-\mathrm{R}$ and $\mathrm{R}-\mathrm{D}$ channel gains of all the $K=3$ users, $M=4$ subband groups, and $J=5$ relays. In the $\mathrm{S}-\mathrm{R}$ links, user $k=0$ benefits from the highest gain of $g_{0,4}^{\mathrm{SR}}=1.42$ through relay $j=4$, user $k=1$ attains the gain of $g_{1,2}^{\mathrm{SR}}=3.07$ using relay $j=2$, whereas user $k=2$ has $g_{2,0}^{\mathrm{SR}}=2.26$ using relay $j=0$. In particular, the sorted CQI set of each $\mathrm{S}-\mathrm{R}$ channel may be obtained by ordering the $g_{k, j}^{\mathrm{SR}}$ values in each row in Table I(a) marked with (1st), (2nd), and (3rd). After choosing the RBs with the best $K$ combination of relays in the rows and the best subband groups in the columns in Table I(a) for transmission over the R-D channels, the user that waits for resource assignment is determined by the corresponding relay, where the specific ordering of $g_{k, j}^{\mathrm{SR}}$ is considered. As a result, compared to the random user assignment of the 
TABLE II

COMPLEXITY COMPARISON OF MU SYSTEMS $(K \leq M)$

\begin{tabular}{l||r|r}
\hline Mode & Proposed Solution & Exhaustive Search \\
\hline \hline$\overline{\text { DRS-DSA }}$ & $\leq \mathcal{O}\{M(J-1)+M \log M\}$ & $\mathcal{O}\left\{M J !+\mathrm{P}_{K}^{M}\right\}$ \\
\hline JDRA-1 & $\leq \mathcal{O}\{J K \log K+M(J-1)+M \log M\}$ & $\mathcal{O}\left\{J K !+M J !+\mathrm{P}_{K}^{M}\right\}$ \\
\hline JDRA-2 & $\leq \mathcal{O}\{K(J-1)+K \log K+J M \log M\}$ & $\mathcal{O}\left\{K J !+K !+J \mathrm{P}_{K}^{M}\right\}$ \\
\hline
\end{tabular}

DRS-DSA scheme, we arrive at the second-hop RBs with indices of $(j, m)=(1,3)$. More explicitly, these RBs are associated with a first-hop quality $g_{2,1}^{\mathrm{SR}}=\mathbf{0 . 5 7}$ for user $k=2$, with the second-hop RB of $(j, m)=(3,1)$ associated with the firsthop quality of $g_{0,3}^{\mathrm{SR}}=0.18$ for user $k=0$, and, finally, with the second-hop $\mathrm{RB}$ of $(j, m)=(0,2)$ associated with the first-hop quality of $g_{1,0}^{\mathrm{SR}}=0.53$ for user $k=1$.

\section{E. FHQA Joint DRA Approach-2}

Rather than finding the best link of a specific user to a relay, the JDRA-2 scheme finds the ordered users, each of which can be supported by a specific relay with the best possible channel quality. We assume that each cooperative relay forwards its $\mathrm{S}-\mathrm{R}$ CQI recorded for each of the $K$ users to the relay with a role of the cooperative cluster head. This head relay can determine the $K$ best $\mathrm{S}-\mathrm{R}$ links from the entire set of $(K J) \mathrm{CQIs}$, and it feeds back this information to the $(J-1)$ relays. Let us now characterize the scheme in more detail as follows.

1) Initialization: Set $\check{\mathcal{J}}=\varnothing, \stackrel{\circ}{\mathcal{K}}=\varnothing, \check{\mathcal{G}}^{\mathrm{SR}}=\varnothing, \stackrel{\circ}{\mathcal{G}}=\varnothing$, and $\stackrel{\circ}{\mathcal{M}}=\varnothing$.

2) First-hop DRS: For the $k$ th user, $\forall k \in \mathcal{K}$.

a) Obtain $\check{j}_{k}=\arg \max _{j \in \mathcal{J}}\left\{g_{k, j}^{\mathrm{SR}}\right\}$.

b) Update $\check{\mathcal{J}}=\check{\mathcal{J}} \cup\left\{\check{j}_{k}\right\}$ and $\check{\mathcal{G}}^{\mathrm{SR}}=\check{\mathcal{G}}^{\mathrm{SR}} \cup\left\{g_{k, \breve{j}}^{\mathrm{SR}}\right\}$.

3) FHQA ordering: For $i=0,1, \ldots, K-1, \forall \check{j}_{k} \in \check{\mathcal{J}}$.

a) Obtain $\stackrel{\circ}{k}_{i}=\arg \max _{k \in \mathcal{K}}\left\{g_{k, \breve{j}}^{\mathrm{SR}}\right\}$.

b) Update $\stackrel{\circ}{\mathcal{K}}=\stackrel{\circ}{\mathcal{K}} \cup\left\{\stackrel{\circ}{k}_{i}\right\}, \stackrel{\circ}{\mathcal{G}} \stackrel{\mathrm{SR}}{=} \stackrel{\circ}{\mathcal{G}} \cup\left\{\begin{array}{c}g_{\circ}^{\mathrm{SR}} \\ k_{i}, \grave{j}\end{array}\right\}, \quad \check{\mathcal{G}}^{\mathrm{SR}}=$ $\check{\mathcal{G}}^{\mathrm{SR}} \backslash\left\{\begin{array}{c}g_{\circ}^{\mathrm{SR}} \\ k_{i}, \check{j}\end{array}\right\}$.

4) Second-hop DSA: For $i=0,1, \ldots, K-1, \forall \check{j}_{k_{i}} \in \check{\mathcal{J}}$.

a) Collect $\mathcal{G}_{\substack{j_{\circ} \\ k_{i}}}^{\mathrm{RD}}=\left\{\underset{\substack{\tilde{j}_{\circ} \\ k_{i}}}{\mathrm{RD}}, g_{\substack{j_{j_{\circ}} \\ k_{i}}}^{\mathrm{RD}}, \ldots, g_{M-1, \grave{j}_{\circ}}^{\mathrm{RD}}\right\}$.

b) Obtain $\stackrel{\circ}{m}_{i}=\arg \max { }_{m \in \mathcal{M}, m \notin \mathcal{M}}\left\{g_{m, \breve{j}_{\circ}}^{\mathrm{RD}}\right\}$.

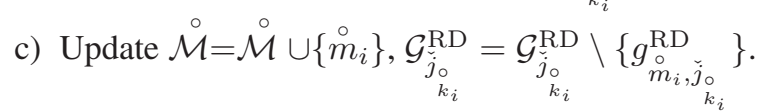

5) User assignment: Allocate the resource pair $\left(\stackrel{\circ}{m_{i}}, \breve{j}_{k_{i}}\right)$ to the user $\stackrel{\circ}{k}_{i}$, for $i=0,1, \ldots, K-1$.

For example, Table $\mathrm{I}(\mathrm{b})$ characterizes the allocation strategy based on the aforementioned FHQA JDRA-2 procedure, which may be contrasted with JDRA-1 in Table I(a). Explicitly, the highest $\mathrm{S}-\mathrm{R}$ channel gain $g_{k, j}^{\mathrm{SR}}$ of each user in the columns in Table I(b) are marked by underlining. Thus, by ordering the underlined values of $g_{j, k}^{\mathrm{SR}}$, JDRA-2 assigns the appropriately ordered users $k=1, k=2$, and $k=0$ to the aforementioned relays of $j=2, j=0$, and $j=4$, respectively. However, the first-hop quality $g_{k, j}^{\mathrm{SR}}$ of JDRA-1 separately determines the order of users at each relay in the rows in Table I(a). By contrast, the ordering of users in JDRA-2 allocates the RB with the highest R-D channel gain $g_{j, m}^{\mathrm{RD}}$ to the specific user at the corresponding relay. These RBs are distinguished by underlining in all subband groups. Observe that some RBs with the highest R-D gains, in fact, may not be considered, such as $g_{1,3}^{\mathrm{RD}}=\mathbf{4 . 4 1}$ and $g_{3,1}^{\mathrm{RD}}=\mathbf{2 . 5 0}$, because the DRS carried in JDRA-2 is based on the first-hop CQI.

\section{F. Comparison of Resource Allocation for OR}

With regard to the pilot-based CSI to be exchanged among the relays, the differences of the FHQA JDRA schemes are related to the amount of the knowledge required with respect to the of S-R links. In particular, the JDRA-1 scheme requires only each relay's ordered set of $K$ users, as determined by their S-R channel gains, whereas the JDRA-2 mode considers the $\mathrm{CQI}$ of all S-R links gleaned from all relay.

As described in Sections III-D and E, both FHQA JDRA schemes have the following three main functions: 1) relay selection; 2) subband allocation; and 3) user assignment. Explicitly, for both JDRA schemes, the subband allocation is based on the second-hop quality, whereas the user assignment depends on the first-hop quality. The relay selection of JDRA-1 is based on the second-hop quality, whereas the relay selection of JDRA-2 relies on the first-hop quality. Therefore, the JDRA-1 method predominantly relies on the second-hop quality, whereas JDRA-2 relies on the first-hop quality. As a result, the attainable performance of both JDRA-1 and JDRA-2 is limited by the quality of its dominant channels.

In addition, the computational complexity of a system that is associated with $K \leqslant M$ and employing various OR-aided resource allocation schemes is presented in Tables II. In particular, the complexity of our proposed JDRA schemes is linearly increased upon increasing $J$. By contrast, for a given $J$, we infer that the $\mathcal{O}$ versus $M$ relationship is linearithmic.

\section{Simulation Results And Discussions}

In this section, the performance achieved by the various resource allocation schemes that rely on DF-aided OR is characterized in the context of channel-coded SC-FDMA systems upon varying the fading channel conditions. The basic assumptions with regard to our channel model and the simulation parameters are summarized in Table III, where diverse multipath fading channels are considered, along with various system configurations. We analyze the attainable performance in terms of the bit error ratio (BER) and energy consumption metrics at a given information rate. 
TABLE III

Simulation PARAMEters

\begin{tabular}{l||r}
\hline Source-to-destination (S-D) fading & No S-D link \\
\hline S-R, R-D fading & Frequency-selective Rayleigh \\
\hline Shadowing & Not considered $\left(\sigma_{\xi}^{2}=0 \mathrm{~dB}\right)$ \\
\hline Path-loss & $G_{\mathrm{SR}}=G_{\mathrm{RD}}=0.5^{-4}$ \\
\hline Modulation & Set-Partition (SP) 4-QAM \\
\hline FEC coding & RSC $(2,1,3), R_{\mathrm{c}}=1 / 2$ \\
\hline Number of subbands per user & $N=12$ \\
\hline Bandwidth expansion factor & $M=6$ \\
\hline Total number of subbands & $U=72$ \\
\hline Number of source users & $K=6$ \\
\hline Number of paths & $L=4$ \\
\hline Transmit power & $P_{k}^{\mathrm{S}}=P_{k}^{\mathrm{R}}=0.5$ \\
\hline Number of iterations & relay: $I_{\mathrm{R}}=0,1, \mathrm{BS}: I_{\mathrm{D}}=1$ \\
\hline
\end{tabular}

\section{A. Energy Efficiency Evaluation}

Let us now evaluate the energy efficiency of both the DT and the proposed OR schemes at the physical layer, when considering the energy consumption due to CQI exchange among relays. ${ }^{6}$ Let $r_{k}^{(\cdot)}, r_{\Sigma}^{(\cdot)}$ denote the coding and modulation rate of the (.)-type transmissions, i.e., that of DT or OR, for the SU and MU scenarios, respectively.

Because we have a block duration of $T_{\mathrm{v}}$ for each RV, as shown in Fig. 4, the $T_{\mathrm{v}}$ pilot RV overhead per RB duration of $N_{\text {rv }} T_{\mathrm{v}}$ may be used for channel estimation for each first- and second-hop transmissions. Hence, we have an overhead-to-data ratio, which is expressed as

$$
\frac{2 T_{\mathrm{v}}}{\left(2 N_{\mathrm{rv}}-2\right) T_{\mathrm{v}}}=\frac{1}{N_{\mathrm{rv}}-1} .
$$

Furthermore, the energy consumption per bit of the DT scheme may be expressed as

$$
\begin{aligned}
\varepsilon_{\mathrm{DT}} & =\frac{\sum_{k=0}^{K-1} P_{k} T}{\sum_{k=0}^{K-1} r_{k}^{\mathrm{DT}}\left(T-N_{\mathrm{rb}} T_{\mathrm{v}}\right)} \\
& =\frac{P_{\Sigma}^{\mathrm{DT}} N_{\mathrm{rv}}}{r_{\Sigma}^{\mathrm{DT}}\left(N_{\mathrm{rv}}-1\right)} \quad(\mathrm{J} / \mathrm{bit})
\end{aligned}
$$

where $T=N_{\mathrm{rb}} N_{\mathrm{rv}} T_{\mathrm{v}}$ denotes the time duration per hop within a frame, including the channel estimation overheads.

By contrast, in addition to the channel estimation overhead, our proposed two-hop FHQA-JDRA-based OR scheme requires further $2 T_{\mathrm{v}} \mathrm{RV}$ s for exchanging the CQI per RB. Hence, we have the total overhead-to-data ratio, which is expressed as

$$
\frac{4 T_{\mathrm{v}}}{\left(2 N_{\mathrm{rv}}-4\right) T_{\mathrm{v}}}=\frac{2}{N_{\mathrm{rv}}-2} \text {. }
$$

Moreover, the corresponding energy consumption per bit of the two-hop OR schemes, including the aforementioned total overheads, may be expressed as

$$
\begin{aligned}
\varepsilon_{\mathrm{OR}} & =\frac{\sum_{k=0}^{K-1}\left(P_{k}^{\mathrm{s}}+P_{k}^{\mathrm{R}}\right) T+\sum_{k=0}^{K-1} P_{k}^{\mathrm{R}}\left(2 N_{\mathrm{rb}} T_{\mathrm{v}}\right)}{\sum_{k=0}^{K-1} 2 r_{k}^{\mathrm{OR}}\left(T-N_{\mathrm{rb}} T_{\mathrm{v}}\right)} \\
& =\frac{P_{\Sigma}^{\mathrm{OR}}\left(N_{\mathrm{rv}}+1\right)}{r_{\Sigma}^{\mathrm{DT}}\left(N_{\mathrm{rv}}-1\right)} \quad(\mathrm{J} / \mathrm{bit})
\end{aligned}
$$

${ }^{6}$ When evaluating the achievable energy efficiency, the half-rate difference imposed by two-hop relaying was, indeed, taken into account in terms of (18). Therefore, we arrived at a fair comparison between the OR and DT scenarios in terms of the ECG formulated in (19). where we have $P_{k}^{\mathrm{S}}=P_{k}^{\mathrm{R}}=0.5 P_{\Sigma} / K$, and $r_{k}^{\mathrm{OR}}=r_{k}^{\mathrm{DT}} / 2=$ $R_{c} Q / 2$.

We define the power reduction $\gamma_{\mathrm{b}}^{\Delta}$ per bit as the SNR reduction per bit, i.e., we have $\gamma_{\mathrm{b}}^{\Delta}=\gamma_{\mathrm{b}}^{\mathrm{DT}}-\gamma_{\mathrm{b}}^{\mathrm{OR}} \mathrm{dB}$, where $\gamma_{\mathrm{b}}^{(\cdot)}$ denotes $E_{b} / N_{0}$ of the (.)-type scheme at a given BER. ${ }^{7}$ Moreover, by using the DT as a reference as defined in [25], the relative ECG of our proposed OR may be expressed as

$$
\mathrm{ECG}=\frac{\varepsilon_{\mathrm{DT}}}{\varepsilon_{\mathrm{OR}}}=\frac{P_{\Sigma}^{\mathrm{DT}} N_{\mathrm{rv}}}{P_{\Sigma}^{\mathrm{OR}}\left(N_{\mathrm{rv}}+1\right)}=\frac{10^{\gamma_{\mathrm{b}}^{\Delta} / 10} N_{\mathrm{rv}}}{N_{\mathrm{rv}}+1} .
$$

Finally, the ERG quantifies the energy savings (in percentage) achieved by a scheme under test with respect to a reference system, yielding

$$
\mathrm{ERG}=100 \times(1-1 / \mathrm{ECG}) \quad \% .
$$

\section{B. BER Performance Over Uncorrelated Fading Channels}

In this section, we evaluate the performance of the resource allocation approaches in Section III, which are employed by the DF-based OR-assisted SC-FDMA uplink. The half-rate RSCcoded SC-FDMA invokes the MMSE turbo FD-LE that relies on the BICM-ID scheme in Fig. 2(b) and (c) at both the relay's and the BS's receivers, where " $I_{\mathrm{R}}=0$ " refers to the classic noniterative FD-LE at the relay.

First, let us consider the BS's receiver using a single antenna. Fig. 5(a) characterizes the DF-relay in Section II-C3 that operates through either hard or soft decisions, denoted as "hardDF" and "soft-DF," respectively, where the BS's turbo receiver invokes a single iteration, ${ }^{8}$ denoted as $I_{\mathrm{D}}=1$. Furthermore, the substantial performance enhancements of the soft-DF schemes may be attained by the turbo FD-LE upon increasing the number of iterations at the relay. This is because carrying out iterative joint equalization and decoding at the relay mitigates the ISI and eliminates the potential error propagation during relaying. In addition, observe the soft-DF curves in Fig. 5(a). The DRS-DSA schemes in Section III-C attain a proved gain of about $1 \mathrm{~dB}$ over the random relay selection (RRS) using the SSA scheme (RRS-SSA) benchmark. Compared to the DRSDSA scheme in Section III-C, at a BER of $10^{-4}$, both the JDRA schemes in Sections III-D and III-E perform similarly and attain an approximately 2 or $1 \mathrm{~dB}$ additional power gain for the $I_{\mathrm{R}}=0$ or $I_{\mathrm{R}}=1$ scenario at the relay's receiver, respectively.

By contrast, the multiple-antenna BS that is invoked in our system offers receive diversity gains. The fading-induced variation of $g_{j, m}^{\mathrm{RD}}$ is beneficially reduced when summing an increased number of $N_{\mathrm{r}}$ independent channel attenuations averaged over the $m$ th subband group for the multipath channel between the

\footnotetext{
${ }^{7} E_{\mathrm{b}}$ denotes the total energy per bit transmitted by the source and the relay per user signal, which is normalized to unity, whereas $N_{0}$ is the noise power spectral density at the receiver, which is assumed to be the same for all the relays and the BS receivers. Hence, $E_{\mathrm{b}} / N_{0}$ represents the average SNR per bit.

${ }^{8}$ Based on our simulation results, using $I_{\mathrm{R}}=1$ iteration at the relay and $I_{\mathrm{D}}=1$ at the BS attains the most substantial incremental power reduction, whereas the power reduction benefits of further iterations becomes more limited compared with the first iteration.
} 


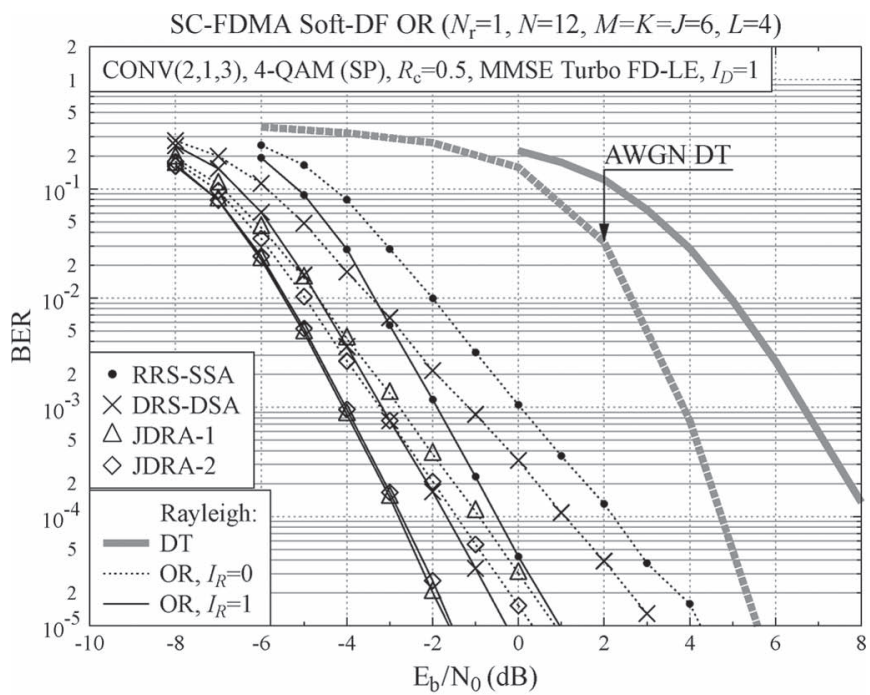

(a)

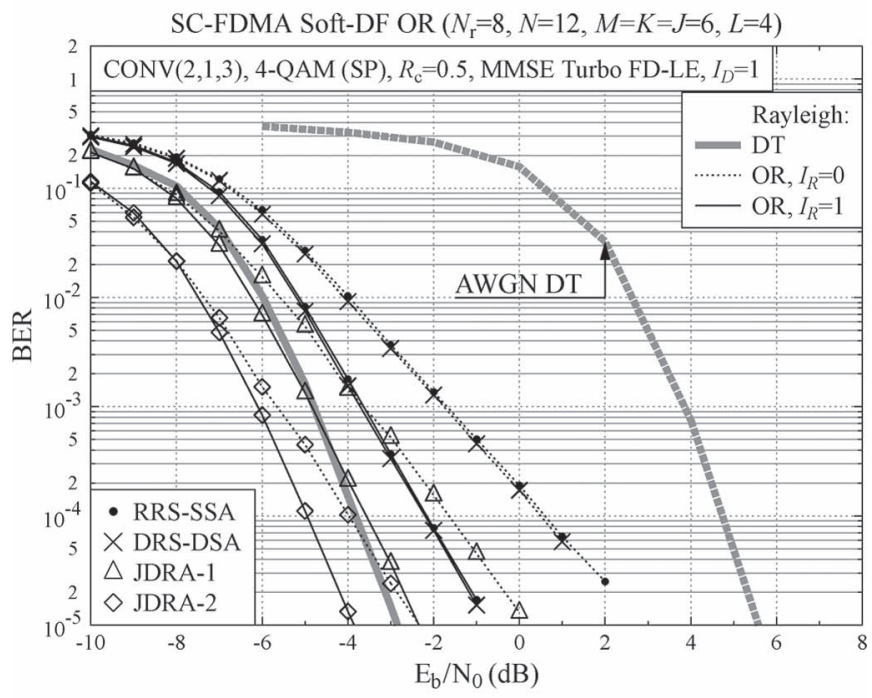

(b)

Fig. 5. BER versus $E_{b} / N_{0}$ performance of the soft-decision-aided DF-based OR-assisted BICM SC-FDMA uplink upon varying the resource allocation schemes when the relay channels experiencing uncorrelated fading. The number of RVs is $N_{\mathrm{rv}}=1$. The BS's receiver invokes the MMSE turbo FD-LE $\left(I_{\mathrm{D}}=1\right)$, whereas the relay's receiver invokes either the classic MMSE FDLE $\left(I_{\mathrm{R}}=0\right)$ or the MMSE turbo FD-LE $\left(I_{\mathrm{R}}=1\right)$. (a) Single-antenna BS's receiver for $N_{\mathrm{r}}=1$ (b) Multiantenna BS's receiver for $N_{\mathrm{r}}=8$.

$j$ th relay and the BS. Therefore, the achievable selection diversity gain of using $J \mathrm{R}-\mathrm{D}$ channels is reduced in the absence of shadowing. As a result, the conventional DRS-DSA schemes in Section III-C has more or less the same performance as the RRS-SSA, which implies that the gain gleaned from selection diversity over R-D channels cannot simply be attained in the multiple-antenna scenario. However, compared to the DRSDSA schemes, the proposed FHQA JDRA methods can achieve an additional gain by rearranging the resources at the ORs by appropriately exploiting the S-R link quality. Importantly, by invoking the JDRA-2 in Section III-E, the first-hop quality becomes the dominant factor in determining the achievable performance benefits of exchanging CQI between the cooperating relays, as discussed in Section III-F. In particular, when we have $N_{\mathrm{r}}=8$ in Fig. 5(b), the proposed JDRA-1 and JDRA-2
TABLE IV

ENERGY-EFFICIENCY OF SOFT-DF BASED OR ASSISTED SC-FDMA USING TURBo RECEIVER FOR $N_{\mathrm{rv}}=6$

\begin{tabular}{l||rr|rr|rr}
\hline & \multicolumn{2}{||}{$\gamma_{\Delta}^{\mathrm{b}}(\mathrm{dB})$} & \multicolumn{2}{|c|}{$E C G$} & \multicolumn{2}{|c}{$E R G(\%)$} \\
$N_{\mathrm{r}}$ & 1 & 8 & 1 & 8 & 1 & 8 \\
\hline \hline DT & 0 & 0 & 1 & 1 & 0 & 0 \\
\hline RRS-SSA & 8.75 & -1.5 & 7.5 & 0.71 & 86.7 & -40.8 \\
\hline DRS-DSA & 10 & -1.5 & 10 & 0.71 & 90 & -40.8 \\
\hline JDRA-1 & 11 & -0.25 & 10.79 & 0.80 & 90.7 & -25.0 \\
\hline JDRA-2 & 11 & 1 & 10.79 & 1.08 & 90.7 & 7.4 \\
\hline
\end{tabular}

schemes that invoke the turbo FD-LE in Fig. 2(b) at both the relay and the BS attain a 1.5- and 3-dB gain over the conventional schemes at a BER of $10^{-4}$, respectively.

\section{Energy Efficiency Over Uncorrelated Fading Channels}

Let us now discuss the energy consumption metrics, i.e., the ECG and ERG in Section IV-A. The attainable power reductions $\gamma_{\Delta}^{\mathrm{b}}$ per bit at a BER of $10^{-4}$ are summarized in Table IV. We evaluated the impact of the channel estimation overhead and the MWR-aided CQI exchange overhead on energy efficiency. In particular, when $N_{\mathrm{r}}=1$, the proposed FHQA JDRA schemes can achieve an ERG of up to $91 \%$ compared to the DT benchmarker. Because the DRS-DSA scheme does not require the CQI exchange overhead, it provides a similar ECG compared to the FHQA JDRA schemes. However, when $N_{\mathrm{r}}=8$, our proposed FHQA-JDRA-2-aided OR scheme attained an ERG of $7.4 \%$, whereas the other methods may not succeed in reducing the transmit power compared to DT, hence resulting in a negative ERG. The reason for this is that multiple-antenna-aided reception provides both a substantial receive diversity gain by exploiting the independent fading of $N_{\mathrm{r}}$ antennas. Therefore, the DRS-DSA method's benefits of assigning the most appropriate resources to MUs erode, and hence, it results in a similar performance to that of the RRS-SSA scheme. In addition, as discussed in Section IV-B, the second-hop quality becomes the dominant factor in determining the performance by invoking the FHQA JDRA-1 approach.

\section{BER Performance Versus the Interleaver Length Over Correlated Fading Channels}

Because both DRS and DSA offer selection diversity gains, we simply adopt a low-complexity RRS combined with the SSA (RRS-SSA) scheme for OR to characterize the advantages of OR and the impact of the interleaver depth $N_{\mathrm{c}}$ on SC-FDMA employing turbo FD-LE for $I_{\mathrm{R}}=I_{\mathrm{D}}=1$. Upon varying $J$ and $f_{\text {nd }}$ for $N_{\text {rv }}=6$, Fig. 6(a) and (b) depicts the BER versus $E_{b} / N_{0}$ performance comparison of soft-DF-based OR systems for interleaver lengths of $N_{\mathrm{c}}=720$ and 7200 coded bits per frame, respectively. In particular, the performance of the singlerelay-aided system for $J=1$ degrades when a fading channel suffers from increased TD correlation associated with a reduced normalized Doppler frequency $f_{\text {nd }}$. This scenario may result in a series of burst errors within a coded bit stream associated with length of $N_{\mathrm{c}}$ over long-term deep fading, where the interleaver with limited depth is no longer effective. However, 


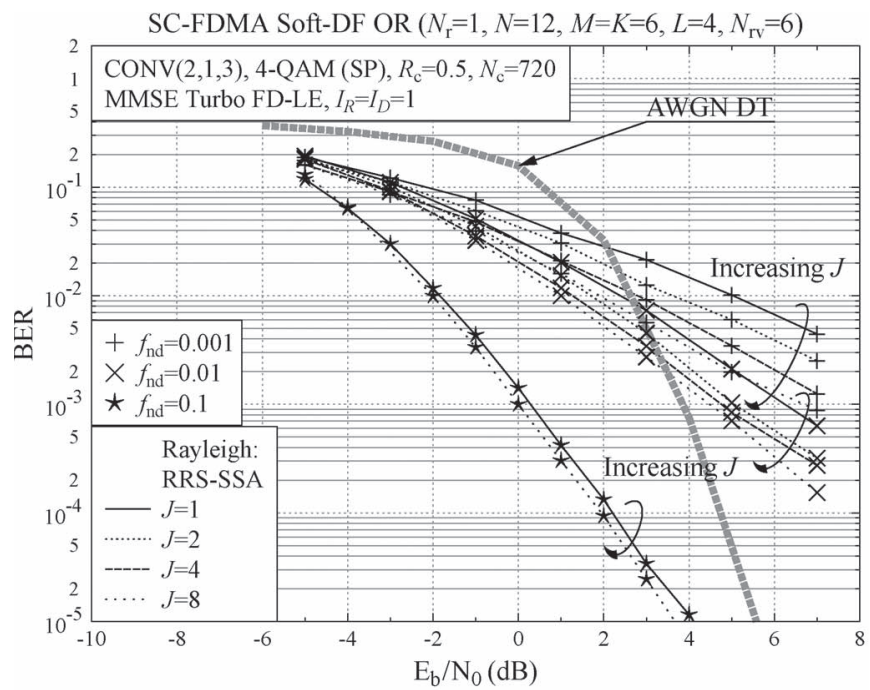

(a)

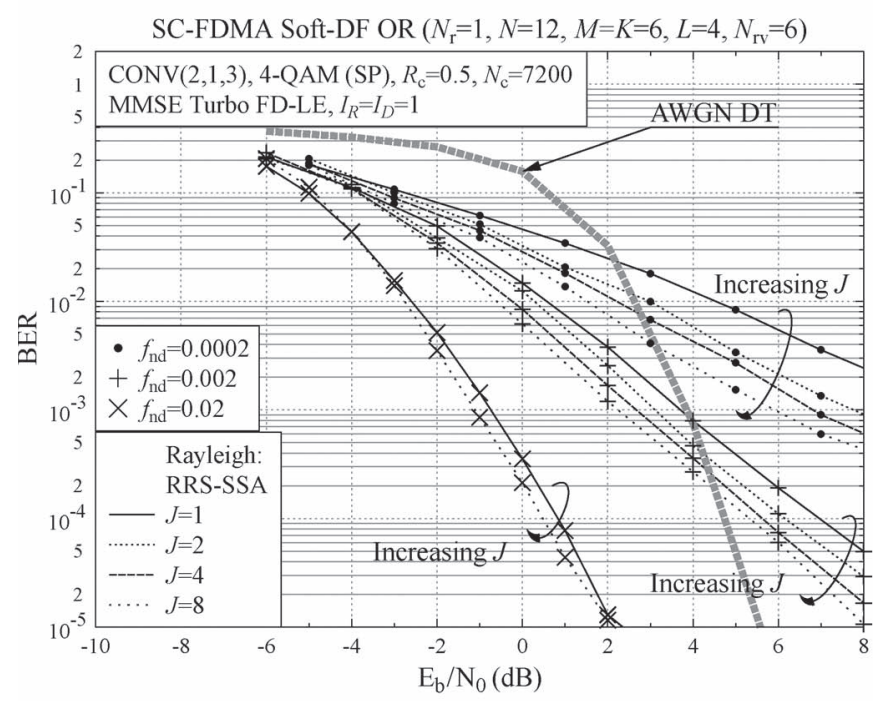

(b)

Fig. 6. BER versus $E_{b} / N_{0}$ performance comparison of RRS-SSA-aided soft-DF-based OR-assisted SC-FDMA uplink upon varying the number of relays $J=1,2,4$, and 8 subject to various normalized Doppler frequencies $f_{\text {nd }}$ when the relay channels experience correlated fading. The number of RVs is $N_{\mathrm{rv}}=6$, and the interleaver length is $N_{\mathrm{c}}=720$ and 7200 coded bits per frame, respectively. (a) $N_{\mathrm{c}}=720$, and $f_{\text {nd }}=0.001,0.01$, and 0.1 . (b) $N_{\mathrm{c}}=7200$, and $f_{\text {nd }}=0.0002,0.002$, and 0.02 .

when increasing the $J$ at a given $f_{\text {nd }}$ and $N_{\mathrm{c}}$, the fading of OR channel is more spatially independent, leading to an improved BER performance, and the transmit energy per bit may be reduced. Therefore, the shortened interleaver-aided DFOR may be considered, particularly on the application subject to limited buffering delay.

\section{CONCLUSION}

In this paper, we have proposed and investigated two novel FHQA JDRA schemes to improve the reliability and energy efficiency of the soft-DF-based OR-assisted SC-FDMA uplink. By exploiting the first-hop quality for the joint design of DRS and DSA at the relays, the proposed FHQA JDRA algorithms outperform the conventional DRS-DSA and achieve up to $2-\mathrm{dB}$ power reduction in channel-coded systems. When using a
SC-FDMA DT benchmark, both FHQA JDRA schemes attain an ERG of $91 \%$ upon invoking a single-antenna BS and an ERG of up to $7.4 \%$ offered by the JDRA-2 scheme in the multiantenna-aided scenario, whereas their counterparts consume significantly more power. Furthermore, to decrease the buffering delay and reduce the transmit power, the interleaver depth of the proposed coded OR systems may be shortened by increasing the number of relays and invoking the soft-DF protocol when communicating over highly correlated fading channels.

\section{REFERENCES}

[1] K. J. R. Liu, A. K. Sadek, W. Su, and A. Kwasinski, Cooperative Communications and Networking. Cambridge, U.K.: Cambridge Univ. Press, 2008.

[2] A. Bletsas, A. Khisti, D. P. Reed, and A. Lippman, "A simple cooperative diversity method based on network path selection," IEEE J. Sel. Areas Commun., vol. 24, no. 3, pp. 659-672, Mar. 2006.

[3] H. G. Myung and D. J. Goodman, Single Carrier FDMA: A New Air Interface for Long Term Evolution. Hoboken, NJ: Wiley, 2008.

[4] F. Khan, LTE for 4G Mobile Broadband: Air Interface Technologies and Performance. Cambridge, U.K.: Cambridge Univ. Press, 2009.

[5] C. Y. Wong, R. S. Cheng, K. B. Letaief, and R. D. Murch, "Multiuser OFDM with adaptive subcarrier, bit and power allocation," IEEE J. Sel. Areas Commun., vol. 17, no. 10, pp. 1747-1758, Oct. 1999.

[6] T. Keller and L. Hanzo, "Adaptive multicarrier modulation: A convenient framework for time-frequency processing in wireless communications," Proc. IEEE, vol. 88, no. 5, pp. 611-640, May 2000.

[7] K. B. Letaief and Y. J. Zhang, "Dynamic multiuser resource allocation and adaptation for wireless systems," IEEE Wireless Commun., vol. 13, no. 4, pp. 38-47, Aug. 2006.

[8] T. Liu, C. Yang, and L.-L. Yang, "A low-complexity subcarrier-power allocation scheme for frequency-division multiple-access systems," IEEE Trans. Wireless Commun., vol. 9, no. 5, pp. 1564-1570, May 2010.

[9] O. Nwamadi, X. Zhu, and A. K. Nandi, "Dynamic physical resource block allocation algorithms for uplink Long Term Evolution," IET Commun., vol. 5, no. 7, pp. 1020-1027, May 2011.

[10] Z. Han, T. Himsoon, W. P. Siriwongpairat, and K. J. R. Liu, "Resource allocation for multiuser cooperative OFDM networks: Who helps whom and how to cooperate," IEEE Trans. Veh. Technol., vol. 58, no. 5, pp. 2378 2391, Jun. 2009.

[11] O. Duval, Z. Hasan, E. Hossain, F. Gagnon, and V. Bhargava, "Subcarrier selection and power allocation for amplify-and-forward relaying over OFDM links," IEEE Trans. Wireless Commun., vol. 9, no. 4, pp. 1293 1297, Apr. 2010.

[12] W. Dang, M. Tao, H. Mu, and J. Huang, "Subcarrier-pair-based resource allocation for cooperative multirelay OFDM systems," IEEE Trans. Wireless Commun., vol. 9, no. 5, pp. 1640-1649, May 2010.

[13] C.-N. Hsu, H.-J. Su, and P.-H. Lin, "Joint subcarrier pairing and power allocation for OFDM transmission with decode-and-forward relaying," IEEE Trans. Signal Process., vol. 59, no. 1, pp. 399-414, Jan. 2011.

[14] J. N. Laneman and G. W. Wornell, "Distributed space-time-coded protocols for exploiting cooperative diversity in wireless networks," IEEE Trans. Inf. Theory, vol. 49, no. 10, pp. 2415-2425, Oct. 2003.

[15] C.-H. Yu and O. Tirkkonen, "Opportunistic multiple relay selection with diverse mean channel gains," IEEE Trans. Wireless Commun., vol. 11, no. 3, pp. 885-891, Mar. 2012.

[16] M. Chen, T. C.-K. Liu, and X. Dong, "Opportunistic multiple relay selection with outdated channel-state information," IEEE Trans. Veh. Technol., vol. 61, no. 3, pp. 1333-1345, Mar. 2012.

[17] T. Wang and L. Vandendorpe, "WSR-maximized resource allocation in multiple-DF-relay-aided OFDMA downlink transmission," IEEE Trans. Signal Process., vol. 59, no. 8, pp. 3964-3976, Aug. 2011.

[18] T. Wang and L. Vandendorpe, "Sum-rate-maximized resource allocation in multiple DF relays aided OFDM transmission," IEEE J. Sel. Areas Commun., vol. 29, no. 8, pp. 1559-1571, Sep. 2011

[19] Y. Jing and H. Jafarkhani, "Single and multiple relay selection schemes and their achievable diversity orders," IEEE Trans. Wireless Commun., vol. 8, no. 3, pp. 1414-1423, Mar. 2009.

[20] J. Zhang, L.-L. Yang, and L. Hanzo, "Energy-efficient channel-dependent cooperative relaying for the multiuser SC-FDMA uplink," IEEE Trans. Veh. Technol., vol. 60, no. 3, pp. 992-1004, Mar. 2011. 
[21] L. Hanzo and B.-J. Choi, "Near-instantaneously adaptive HSDPA-style OFDM versus MC-CDMA transceivers for WIFI, WIMAX, and nextgeneration cellular systems," Proc. IEEE, vol. 95, no. 12, pp. 2368-2392, Dec. 2007.

[22] K. C. Lee and L. Hanzo, "MIMO-assisted hard versus soft decodingand-forwarding for network coding aided relaying systems," IEEE Trans. Wireless Commun., vol. 8, no. 1, pp. 376-385, Jan. 2009.

[23] Y. Li, B. Vucetic, T. F. Wong, and M. Dohler, "Distributed turbo coding with soft information relaying in multihop relay networks," IEEE J. Sel. Areas Commun., vol. 24, no. 11, pp. 2040-2050, Nov. 2006.

[24] D. Gunduz, A. Yener, A. J. Goldsmith, and H. V. Poor, "The multiway relay channel," in Proc. IEEE ISIT, Jul. 2009, pp. 339-343.

[25] C. Han, T. Harrold, S. Armour, I. Krikidis, S. Videv, P. M. Grant, H. Haas, J. S. Thompson, I. Ku, C.-X. Wang, T. A. Le, M. R. Nakhai, J. Zhang, and L. Hanzo, "Green radio: Radio techniques to enable energy-efficient wireless networks," IEEE Commun. Mag., vol. 49, no. 6, pp. 46-54, Jun. 2011.

[26] M. Tüchler and A. C. Singer, "Turbo equalization: An overview," IEEE Trans. Inf. Theory, vol. 57, no. 2, pp. 920-952, Feb. 2011.

[27] L. Hanzo, T. Liew, B. Yeap, R. Tee, and S. X. Ng, Turbo Coding, Turbo Equalization and Space-Time Coding (EXIT-Chart-Aided Near-Capacity Designs for Wireless Channels). Hoboken, NJ: Wiley, 2011.

[28] L.-L. Yang, Multicarrier Communications. Hoboken, NJ: Wiley, 2009.

[29] L. Hanzo, M. Münster, B.-J. Choi, and T. Keller, OFDM and MC-CDMA for Broadband Multiuser Communications, WLANs and Broadcasting. New York: Wiley, 2003.

[30] M. Tüchler, R. Koetter, and A. C. Singer, "Turbo equalization: Principles and new results," IEEE Trans. Commun., vol. 50, no. 5, pp. 754-767, May 2002.

[31] M. Tüchler, A. C. Singer, and R. Koetter, "Minimum mean square error equalization using a priori information," IEEE Trans. Signal Process., vol. 50, no. 3, pp. 673-683, Mar. 2002.

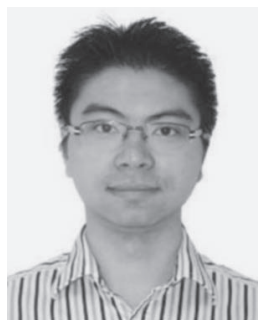

Jiayi Zhang ( $\left.\mathrm{S}^{\prime} 07\right)$ received the B.Eng. degree in information engineering from Nanjing University of Aeronautics and Astronautics, Nanjing, China, in 2006 and the M.Sc. degree (with distinction) in wireless communications and the Ph.D. degree in electronics and electrical engineering from the University of Southampton, Southampton, U.K., in 2007 and 2012, respectively.

During his Ph.D. research within the School of Electronics and Computer Science, University of Southampton, he was also involved with the Virtual Centre of Excellence in Mobile and Personal Communications, where he worked on the Core 4 Delivery Efficiency and Core 5 Green Radio Projects. Since August 2012, he has been with the Alcatel-Lucent Shanghai Bell, Shanghai, China, where he is currently a Research Scientist. His research interest is focused on wireless communications, including energyefficient cooperative relaying, multicell cooperation, resource allocation, and transceiver signal processing for single-carrier and multicarrier broadband transmission systems. Details about his publications can be found at http://uk.linkedin.com/in/jiayizhang/.

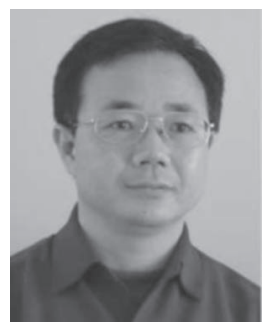

Lie-Liang Yang (M'98-SM'02) received the B.Eng. degree in communications engineering from Shanghai Tiedao University, Shanghai, China, in 1988 and the M.Eng. and Ph.D. degrees in communications and electronics from Northern (Beijing) Jiaotong University, Beijing, China, in 1991 and 1997, respectively.

From June 1997 to December 1997, he was a Visiting Scientist with the Institute of Radio Engineering and Electronics, Academy of Sciences of the Czech Republic, Prague, Czech Republic. Since December 1997, he has been with the University of Southampton, Southampton, U.K., where he is currently a Professor with the School of Electronics and Computer Science. He has published more than 240 research papers in journals and conference proceedings, has been the author or a coauthor of three books, and has published several book chapters. Details about his publications can be found at http://www-mobile.ecs.soton.ac.uk/lly/. He is currently an Associate Editor for the Journal of Communications and Networks and the Security and Communication Networks Journal. His research interests include wireless communications, networking, and signal processing.

Dr. Yang is currently an Associate Editor for the IEEE TRANSACTIONS ON VEHICULAR TECHNOLOGY.

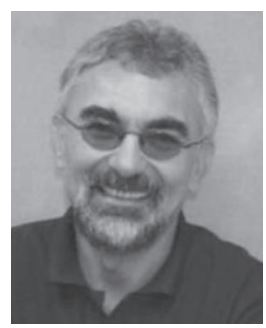

Lajos Hanzo (F'08) received the Master degree, the $\mathrm{Ph} . \mathrm{D}$. degree, and the Honorary Doctorate "Doctor Honaris Causa" degree, all from the Technical University of Budapest, Budapest, Hungary, in 1976, 1983, and 2009, respectively.

During his 35-year career in telecommunications, he has held various research and academic posts in Hungary, Germany, and the U.K. Since 1986, he has been with the School of Electronics and Computer Science, University of Southampton, Southampton, U.K., where he is currently the Chair in Telecommunications. He has successfully supervised $80 \mathrm{Ph} . \mathrm{D}$. students, has been a coauthor of 20 John Wiley/IEEE Press books on mobile radio communications totaling in excess of 10000 pages, and presented keynote lectures. He is currently directing a 100-strong academic research team, working on a range of research projects in wireless multimedia communications sponsored by industry, the Engineering and Physical Sciences Research Council (EPSRC) U.K., the European IST Program, and the Mobile Virtual Centre of Excellence (VCE), U.K. He is an enthusiastic supporter of industrial and academic liaison and offers a range of industrial courses. Since 2009, he has also been a Chair Professor with Tsinghua University, Beijing, China. For further information on research in progress and associated publications, see http://wwwmobile.ecs.soton.ac.uk/.

Dr. Hanzo is a Governor of the IEEE Vehicular Technology Society. He has served as the Technical Program Committee (TPC) and General Chair of IEEE conferences, published more than 1250 research entries on IEEE Xplore, and received a number of distinctions. From 2008 to 2012, he was the Editor-inChief of the IEEE Press. He is a Fellow of the Royal Society of Engineering, the IET, and of EURASIP. 